\title{
Maturation of Neuronal Form and Function in a Mouse Thalamo-Cortical Circuit
}

\author{
Richard A. Warren and Edward G. Jones \\ Department of Anatomy and Neurobiology, University of California, Irvine, California 92697, and Neural Systems \\ Laboratory, Frontier Research Program in Brain Mechanisms of Mind and Behavior, The Institute for Physical and \\ Chemical Research, Wako, Saitama 351-01, Japan
}

Postnatal development of physiological properties underlying slow intrathalamic oscillations was studied by whole-cell recording from synaptically coupled neurons of the reticular nucleus (RTN) and ventral posterior nucleus (VPN) of mouse brain slices in vitro and compared with the morphological development of dye-injected cells. Between postnatal days 3 and 11 (P3-P11), progressive changes in RTN and VPN neurons included shortening of the membrane time constant, decreasing input resistance, and lowering of the resting membrane potential (RMP). Low-threshold $\mathrm{Ca}^{2+}$ spikes (LTS) were present from P3, but their capacity to sustain multispike bursts was limited before P11. Synaptic responses were evoked in RTN and VPN neurons by electrical stimulation of the internal capsule from P3. Younger RTN neurons responded with a single spike, but their capacity to fire bursts gradually improved as the RMP reached levels below the LTS activation potential. Concomi- tantly, as the reversal potential of the inhibitory postsynaptic potential in VPN neurons became more negative, its capacity to deinactivate the LTS increased, and rebound bursts that could maintain oscillations were produced; sustained oscillations became the typical response to internal capsule stimulation at $\mathrm{P} 12$. The functional maturation of the intrathalamic circuitry, particularly between P10 and P14, occurs in parallel with the morphological maturation (size, dendritic growth, and dendritic field structure) of individual RTN and VPN neurons, as studied by confocal microscopy. Maturation of RTN cells led that of VPN cells by 2-3 d. The appearance of intrathalamic oscillations is probably correlated with the appearance of slow-wave sleep in postnatal animals.

Key words: reticular nucleus; ventral posterior nucleus; whole-cell recording; oscillations; injected cells; sleep
In adult animals and humans, the cortical electroencephalogram (EEG) shows typical patterns of activity closely correlated with the sleep-waking cycle. During periods of wakefulness and paradoxical sleep, the amplitude of the cortical EEG is small and the EEG is desynchronized because of the asynchronous, tonic firing of cortical and thalamic neurons (Steriade et al., 1990). By contrast, during periods of drowsiness and the early stages of sleep, the EEG displays slow waves of large amplitude that occur with a frequency of 7-14 Hz.

By contrast with adults, the cortical EEG of neonatal mammals displays only patterns characteristic of wakefulness and paradoxical sleep, with very little of the synchronization typical of slowwave sleep (Jouvet-Mounier et al., 1970; Domich et al., 1987). It appears as if there is direct passage from wakefulness to paradoxical sleep without the intermediate stages of slow-wave sleep. In humans, EEG spindles are absent during the first 2 months of life, and the EEG pattern becomes adult-like at $\sim 14$ years of age (Sterman, 1972; Harper, 1983). In rodents, the adult pattern is observed at $\sim 30 \mathrm{~d}$ of age. In the rat, there is an almost total absence of slow-wave EEG during the first 10 to 12 postnatal (P)

Received July 7, 1996; revised Oct. 10, 1996; accepted Oct. 14, 1996.

This project was supported by National Institutes of Health Grant NS 30109, the U.S. Public Health Service, and the Frontier Research Program. R.A.W. was supported by the Medical Research Council of Canada. We are grateful to Drs. T. Hashikawa and X.-B. Liu for their help in filling and imaging thalamic neurons.

Correspondence should be addressed to Dr. Edward G. Jones, Department of Anatomy and Neurobiology, University of California, College of Medicine, Irvine, CA 92697.

Dr. Warren's present address: Centre de Recherche Fernand-Seguin, 7331 Rue Hochelaga, Montréal, Québec, Canada H1N 3V2.

Copyright (C) 1996 Society for Neuroscience $0270-6474 / 96 / 170277-19 \$ 05.00 / 0$ days (Jouvet -Mounier et al., 1970), even though thalamo-cortical connections through which spindle waves are imposed on the cortex are present from at least day 3 in mice (Agmon and O'Dowd, 1992) and probably from a similar age in rats (Catalano et al., 1995). These observations suggest that thalamic circuitry and/or the properties of thalamic neurons that underlie spindling and slow waves in the EEG are immature at birth and develop over a relatively protracted postnatal period.

Generation of spindle oscillations in the thalamus depends largely on the physiological characteristics of thalamic neurons, notably their capacity to discharge rebound, low-threshold $\mathrm{Ca}^{2+}$ spikes overridden by bursts of $\mathrm{Na}^{+} / \mathrm{K}^{+}$action potentials and on synaptic interactions between the reticular nucleus (RTN) of the thalamus and thalamo-cortical relay neurons that maintain the rhythmicity (Steriade et al., 1985, 1993; von Krosigk et al., 1993; Huguenard and Prince, 1994; Warren et al., 1994; Bal et al., 1995a,b). Lack of spindle waves at early ages could depend on immaturity of or lack of synaptic connectivity between these thalamic elements or on immaturity of the membrane characteristics that underlie the generation of bursts of action potentials responsible for both spindling and synchronizing the RTN-relay neuron circuit. We have used an in vitro mouse thalamo-cortical preparation (Agmon and Connors, 1991), in which spindle-like oscillations can be evoked by stimulation of the internal capsule in P12 and older animals (Warren et al., 1994), to study postnatal development of the physiological characteristics of the circuit linking the RTN and the ventral posterior nucleus (VPN) in the period before $\mathrm{P} 12$. The physiological observations were correlated with studies of the morphological development of these cells up to 
P21, as seen by intracellular injection of dye and confocal microscopy. Preliminary results have appeared in abstract form (Warren and Jones, 1994, 1995).

\section{MATERIALS AND METHODS}

Physiology. The methods were the same as those used on in vitro slices from P12-P21 mice (Warren et al., 1994). ICR mice (Harlan Sprague Dawley), 3- to 11-d-old (P3-P11) were anesthetized either by hypothermia (P3-P5) or with halothane (P6-P11) and decapitated. The brain was quickly removed and chilled in artificial CSF (ACSF) containing (in mM): $\mathrm{NaCl} 126, \mathrm{KCl} 3, \mathrm{NaH}_{2} \mathrm{PO}_{4} 1.25, \mathrm{MgSO}_{4} 1.3, \mathrm{CaCl}_{2} 2.5, \mathrm{NaHCO}_{3} 26$, and dextrose $20, \mathrm{pH} 7.4$, when bubbled with $95 \% \mathrm{O}_{2} / 5 \% \mathrm{CO}_{2}$. All chemicals were obtained from Sigma (St. Louis, MO). Slices (400- $\mu$ m-thick) comprising VPN, RTN, and somatosensory cortex were cut on a vibratome in the oblique plane of Agmon and Connors (1991), which preserves thalamocortical and corticothalamic connectivity from at least P0-P21 (Agmon and O'Dowd, 1992; Agmon et al., 1993, 1995; Warren et al., 1994) and transferred to a submerged-type recording chamber (Medical Systems, Greenvale, NY). Experiments were performed at room temperature $\left(22-25^{\circ} \mathrm{C}\right)$, and ACSF aerated with $95 \% \mathrm{O}_{2} / 5 \% \mathrm{CO}_{2}$ was superfused at a rate of $1.5-2 \mathrm{ml} / \mathrm{min}$ throughout the experiments. We shall describe the results in a continuous sequence from P3 to P21 by incorporating data obtained from P12-P21 slices in the course of the previous study.

Whole-cell recording pipettes had a resistance of 5 to $12 \mathrm{M} \Omega$ when filled with an internal solution containing (in $\mathrm{mM}$ ): potassium gluconate 140, $\mathrm{MgCl}_{2} 2, \mathrm{CaCl}_{2}$ 0.1, EGTA/KOH 1.1, HEPES 10, $\mathrm{K}_{2}$-ATP 2, $\mathrm{Na}_{2}-$ GTP $0.5, \mathrm{pH} 7.3 \pm 0.05$, adjusted with $\mathrm{KOH}$ and with an osmolarity of $275 \pm 5$ mOsm. Signals were recorded with an Axoclamp-2A amplifier (Axon Instruments, Foster City, CA) using bridge mode and continuous single-electrode voltage clamp (SEVC) mode for current- and voltageclamp recordings, respectively. During SEVC, the membrane potential was clamped within $1 \mathrm{mV}$ of the actual resting membrane potential (RMP) to prevent a shift in the reversal of $\mathrm{Cl}^{-}$-mediated inhibitory postsynaptic currents (IPSCs) (Zhang et al., 1991). The low pass filter was set at $3 \mathrm{kHz}$, and gain was set at $70-300 \mathrm{pA} / \mathrm{mV}$ during SEVC. The access resistance of cells, measured by bridge-balance, was almost invariably in the range of 8 to $15 \mathrm{M} \Omega$ and never exceeded $20 \mathrm{M} \Omega$. The offset potential was compensated to $0 \mathrm{mV}$ immediately before breaking into the cell, and RMP was measured within seconds of breaking in. The offset potential (usually $<5 \mathrm{mV}$ ), measured on withdrawal from a cell, was subtracted from the RMP as remeasured just before withdrawal. This usually gave a value within $2 \mathrm{mV}$ of the initial measurement. The liquid junctional potential was not measured and, thus, not subtracted from measurements of membrane potential. It can be assumed to be $\sim 10 \mathrm{mV}$, based on reports in which a similar internal solution was used (Huguenard and Prince, 1992; Spigelman et al., 1992). Unstable recordings were rejected. Data were digitized via a CED 1401 interface (Cambridge Electronic Design, Cambridge, UK) at $1-10 \mathrm{kHz}$ and analyzed using CED software packages.

Input resistance $\left(R_{\text {in }}\right)$, membrane time constant $\left(\tau_{\mathrm{m}}\right)$, and action potential amplitude were measured using standard methods as described previously (Warren et al., 1994). The width of action potentials was measured at half amplitude with a resolution of $0.1 \mathrm{msec}$.

Synaptic responses were evoked in VPN and RTN neurons by electrical stimulation of the internal capsule, using a monopolar tungsten microelectrode. The stimulus consisted of a cathodal voltage pulse of $0.1 \mathrm{msec}$ delivered at $0.05-0.1 \mathrm{~Hz}$. IPSP and IPSC reversal potentials in VPN neurons were estimated from voltage-voltage $\left(V_{\mathrm{R}}-V_{\mathrm{m}}\right)$ or current-voltage $\left(I_{\mathrm{R}}-V_{\mathrm{m}}\right)$ relationships obtained by synaptic activation during square current or voltage pulses in bridge or SEVC mode, respectively.

Drugs used included (in $\mu \mathrm{M}$ final concentration): bicuculline methiodide (BMI) 2.5-10, D-2-amino-5-phosphonovaleric acid (APV) 50, and 6-cyano-7-nitroquinoxaline-2,3-dione (CNQX) 20, all obtained from RBI (Natick, MA).

Statistical analysis was performed using SigmaStat software (Jandel Scientific, San Rafael, CA). Parametric tests were used if the data met the assumptions of the appropriate test. When the assumptions of the test were not fulfilled, these were usually met after logarithmic or square root transformation of the data, and the tests were performed on transformed data. If the assumptions could not be met after transforming the data, then nonparametric tests were used. Values are presented as averages \pm SEM.

Morphology. To obtain a large and consistently well-labeled sample of RTN and VPN cells for morphological analysis during the period of maturation of physiological properties, cells were injected with dye in fixed slices. Mouse pups at P3, P7, P10, P14, and P21 were perfused through the heart with saline followed by a fixative mixture of $0.1 \%$ glutaraldehyde and $4 \%$ paraformaldehyde in $0.1 \mathrm{M}$ phosphate buffer, $\mathrm{pH}$ 7.4. The brain was removed and post-fixed in the same fixative at $4^{\circ} \mathrm{C}$ for $12-72 \mathrm{hr}$. Slices ( $400-\mu \mathrm{m}$-thick) slices were cut on a vibratome in the same plane as fresh tissue (see above), collected in cold phosphate buffer, and stored at $4^{\circ} \mathrm{C}$ for $12-48 \mathrm{hr}$. Selected slices were immunocytochemically stained for GABA using a rabbit anti-GABA antiserum (Sigma) and fluorescein-labeled secondary immunoglobulins, as described previously (Jones et al., 1994).

A slice was transferred to a glass microscope slide and covered with a piece of filter paper containing a small opening sufficient to expose VPN and RTN and transferred to the stage of an epifluorescence microscope (Nikon, Tokyo, Japan) equipped with long-working-distance objectives. A glass micropipette filled with a 3-5\% aqueous solution of Lucifer yellow (Aldrich, Milwaukee, WI) and attached to a micromanipulator was used to impale and inject single neurons under direct visual guidance. The pipette was advanced slowly in the tissue while giving small and short periodic pulses of negative direct current just sufficient to produce a barely visible cloud-like ejection of the dye under epifluorescence. Successful impalement was indicated by accumulation of the dye in a soma and its propagation throughout the processes of the cell. The ejecting current was increased to speed up the filling process. After filling several neurons, the slice was post-fixed in $4 \%$ paraformaldehyde for $1 \mathrm{hr}$ and stored in $0.1 \mathrm{M}$ phosphate buffer at $4^{\circ} \mathrm{C}$.

Slices were washed three times for $5 \mathrm{~min}$ each in cold phosphate buffer, dehydrated in graded alcohols, and cleared in methyl salicylate for 20 $\mathrm{min}$. The slices were mounted in methyl salicylate between two coverslips, and the most completely filled neurons were scanned in serial focal planes through the cell, using an inverted epifluorescence microscope and a BioRad MRC 600 (Cambridge, MA) laser confocal system. Final images of filled neurons consisted of projections of 20-50 planar images separated by $1-2.5 \mu \mathrm{m}$. Measurements of the somata of filled RTN and VPN neurons were made on $10 \times 15 \mathrm{~cm}$ prints of the images, using a Eutectic Neuron Tracing System (Raleigh, NC). The somatic attributes measured include the area and perimeter. From these measures, the program calculates the diameter of the soma as if it were a perfect circle, using the formula:

$$
\text { mean diameter }=\sqrt{(4 \times \text { area } / \pi)},
$$

and provides a form factor, representing a measure of "roundness" which is 1 for a circular soma and 0.1 for a flat, cigar-shaped soma, calculated by the formula:

$$
\text { form factor }=4 \times \pi \times \text { area/perimeter }{ }^{2}
$$

Using the same image prints, the number of dendrites in the best filled neurons that crossed concentric circles separated by $15 \mu \mathrm{m}$ starting at the center of the soma were counted to evaluate quantitatively the branching pattern of RTN and VPN neurons (Sholl, 1953). The extent of the dendritic field was also measured and its general shape characterized according to the orientation of the dendrites.

\section{RESULTS}

\section{Membrane properties}

Whole-cell recordings were made from 23 RTN and 31 VPN neurons in slices from P3-P11 mice. Results obtained for these neurons were added to data previously obtained from 13 RTN and 38 VPN neurons recorded in slices from animals aged P12P21 (Warren et al., 1994) to give a continuous description of the maturation of the system. Basic membrane characteristics of both RTN and VPN neurons changed significantly during the first two postnatal weeks. The rate of change tapered off toward the end of the second postnatal week, at which time oscillatory behavior became evident in the interconnected RTN-VPN network. Although the properties of both RTN and VPN neurons changed dramatically during the period before P12, certain of the specific features that characterize these neurons in older animals were already present and could be used to distinguish them at the youngest ages. The changes observed during the early postnatal 
period appeared as a smooth maturation of these characteristics rather than as a dramatic change in fundamental properties.

Basic membrane properties of RTN and VPN neurons as a function of postnatal age are illustrated in Figure $1 A-C$. In both RTN and VPN neurons, the RMP appeared relatively depolarized in animals $<1$ week old, especially in the RTN neurons (Fig. $1 A$ ). The RMP became progressively more negative in both RTN and VPN neurons from P3 to P10. Beyond this point, the RMP of VPN neurons stabilized at approximately $-59 \mathrm{mV}$, whereas that of RTN neurons became significantly more negative, approaching $-63 \mathrm{mV}$, in animals $\geq 2$ weeks of age.

During the entire period studied, the membrane time constant $\left(\tau_{\mathrm{m}}\right)$ of RTN neurons was on average about half that of VPN neurons and, in both types of neurons, it decreased by $\sim 50 \%$ during the period from P3 to P4 until the end of second postnatal week (Fig. $1 B$ ). The change occurred gradually throughout that period, after which the $\tau_{\mathrm{m}}$ of both RTN and VPN neurons remained virtually unchanged for the remainder of the period studied.

The input resistance $\left(R_{\mathrm{in}}\right)$ of both RTN and VPN neurons decreased significantly from P3 to P12, following a pattern of change similar to that of $\tau_{\mathrm{m}}$ (Fig. 1C). The $R_{\mathrm{in}}$ of VPN neurons decreased more than twofold from P5 to P12, after which it remained unchanged to $\mathrm{P} 21$. The $R_{\text {in }}$ of RTN neurons followed a similar pattern, but despite the large changes in $R_{\text {in }}$, at all ages RTN neurons displayed smaller $R_{\text {in }}$ than VPN neurons; that is, the changes occurred in parallel, and RTN and VPN neurons displayed these distinguishing characteristics throughout the period studied.

The changes in $\tau_{\mathrm{m}}$ and $R_{\mathrm{in}}$ were apparent in the voltage responses produced by intracellular injection of positive and negative current pulses in both RTN and VPN (Fig. 2) neurons. Typically, the $(I-V)$ relationship in RTN neurons was linear along a wide range of membrane potential (e.g., Fig. $2 B, C$ ). In some of the youngest RTN neurons (Fig. $2 A$ ), the $I-V$ curve was S-shaped, suggesting the presence of an inward rectifying conductance in young RTN neurons that attenuated over time. The opposite pattern was observed in VPN neurons, because the $I-V$ relationship could be linear over a relatively wide range of voltages in neurons $<$ P7 (Fig. 2D). More commonly, inward rectification was observed with hyperpolarizations of $>20 \mathrm{mV}$ and an even more pronounced outward rectification, with depolarizing current pulses resulting in S-shaped $I-V$ curves (Fig. $2 E, F$ ). The inward and outward rectifications observed in VPN neurons bore a close resemblance to those reported in thalamic relay cells of other species (Jahnsen and Llinás, 1984; McCormick and Pape, 1991; Soltesz et al., 1991).

\section{Discharge properties}

As with basic membrane properties, the firing properties of RTN and VPN neurons were already differentiated at the earliest ages studied. At all ages, RTN action potentials were faster than VPN action potentials, but no difference in the amplitude of the action potential was detected (Fig. $1 D, E$ ). At P3, RTN action potentials were already followed by a much larger after hyperpolarization than VPN action potentials, and this distinction was present from P3 to P21. Another characteristic that was present throughout the period studied in VPN neurons was a slow depolarizing ramp that usually preceded tonic firing of the neuron during current injection, as reported previously in guinea pig lateral geniculate neurons (McCormick, 1991). In comparison, firing in RTN neurons in response to depolarizing current pulses was never preceded by a depolarizing ramp.

During the days after P4, the firing characteristics of RTN and VP neurons matured; the most notable change was the shortening of the action potential duration (Fig. $1 D$ ). There was also a slight increase in the amplitude of the action potential that could probably be attributed to the decrease in RMP (Fig. 1E). The spiking characteristics stabilized by P12 and did not change significantly thereafter.

The spike trains of both RTN and VPN neurons were regular; cells repolarized almost completely between action potentials, and there was little frequency adaptation at all postnatal ages studied (Fig. $2 A, B, D, E$ ). The tonic firing frequency as a function of the current injected was examined in both RTN and VPN neurons up to P11 (Fig. $3 A, B$ ). The frequency curves of both RTN and VPN neurons moved to the right during postnatal development, showing that more current was needed to induce firing in older neurons (Fig. $3 A, B$ ). In addition, the slope of the frequency curves decreased significantly during the period studied (Fig. $3 C, D$ ), and a statistically significant correlation was found between the slope of the frequency curves and postnatal age for both RTN $\left(r^{2}=0.419\right.$, $p=0.017, n=13)$ and VPN $\left(r^{2}=0.445, p=0.005, n=16\right)$ neurons. Thus, more current was needed to produce a change in firing frequency in older than in younger VPN and RTN neurons, and more current was needed to reach similar firing frequencies in older neurons than in younger neurons. At all ages, there were also marked differences between RTN and VPN neurons. The slope of the frequency curves of RTN neurons was on average much smaller than that of VPN neurons $(0.168 \pm 0.027 \mathrm{~Hz} / \mathrm{pA}$ and $0.326 \pm 0.041 \mathrm{~Hz} / \mathrm{pA}$, respectively; $t s=-3.654$, df $=27, p=$ 0.001) (Fig. 3C,D).

Low-threshold $\mathrm{Ca}^{2+}$ depolarizations could be readily produced in both RTN and VPN neurons by application of current pulses at all ages studied (Figs. 2, 4), but their capacity to sustain bursts of $\mathrm{Na}^{+} / \mathrm{K}^{+}$spikes was limited in the younger animals and was often absent in VPN neurons. In RTN neurons P11 and younger, the majority $(76 \% ; 16 / 21)$ fired more than one $\mathrm{Na}^{+}-\mathrm{K}^{+}$spike when the low-threshold spike (LTS) was deinactivated, but bursts consisting of more than three $\mathrm{Na}^{+}-\mathrm{K}^{+}$spikes were consistently observed only in P10 and P11 neurons and never exceeded four, even under conditions that maximally activated the LTS (Fig. $4 A-C)$. In the remaining RTN neurons younger than P11 (24\%; $5 / 21$ ), only one $\mathrm{Na}^{+}-\mathrm{K}^{+}$spike could be elicited. At all ages, the character of the bursts was similar whether produced by passive membrane repolarization after a hyperpolarizing current pulse or by a depolarizing pulse from a hyperpolarized membrane potential. Bursting in RTN neurons was usually very stereotypic in the sense that once initiated, the number of $\mathrm{Na}^{+} / \mathrm{K}^{+}$spikes was not much influenced by the size of the depolarization or the membrane potential from which it was elicited. This is illustrated in Figure $4, B$ and $C$, in which two bursts (arrows) elicited by the same current pulse but at different membrane potentials elicited the same number of $\mathrm{Na}^{+} / \mathrm{K}^{+}$spikes; in both examples, one was evoked just above threshold and was preceded by a slow depolarization lasting several milliseconds (right arrow in Fig. 4B,C), whereas the other lacked the slow depolarization and was much more readily elicited by the current pulse (left arrow in Fig. 4B,C).

By contrast with RTN neurons, the vast majority of VPN neurons at P11 and younger $(79 \% ; 23 / 29)$ fired none $(n=8)$ or a single action potential $(n=15)$ under conditions in which the LTS was maximally activated (Fig. $4 D, E$ ). More than one action potential riding on the LTS was observed for the first time only at P7, 


\section{A- Resting Membrane Potential}

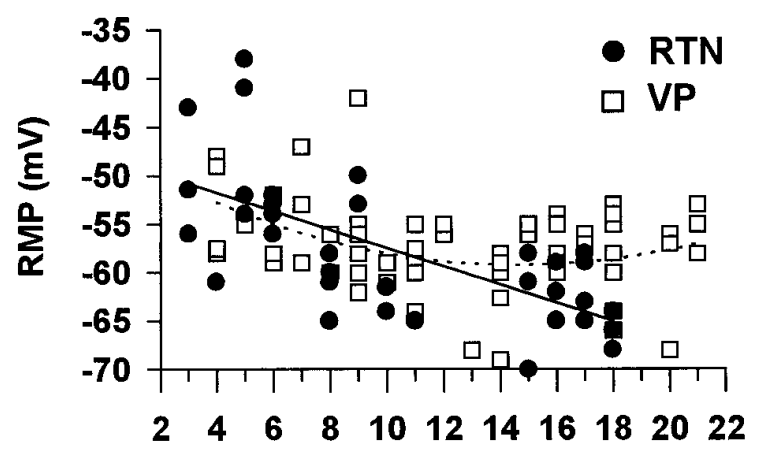

B- Time Constant

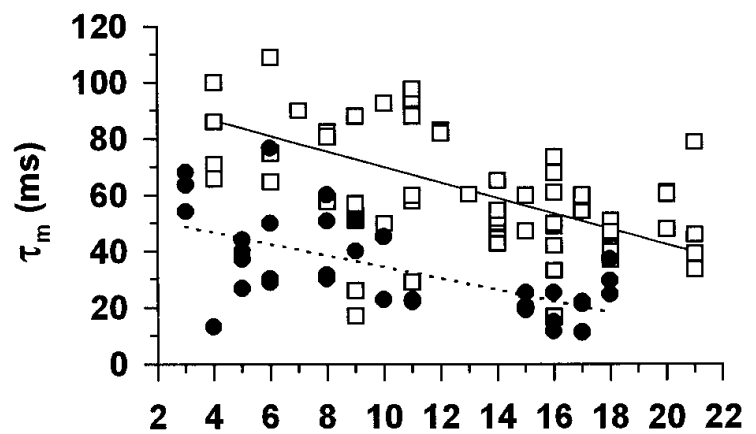

C- Input Resistance

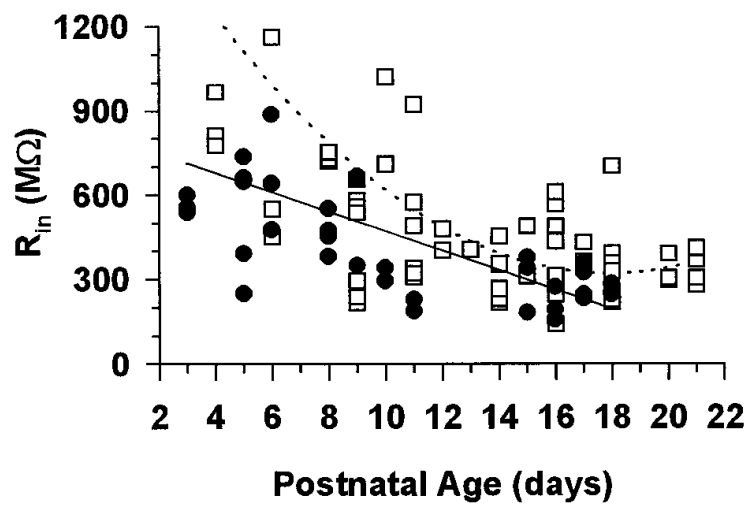

D- Action Potential Duration

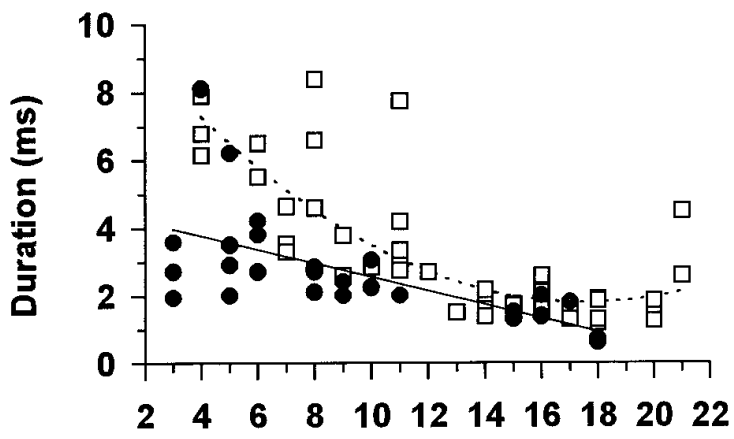

E- Action Potential Amplitude

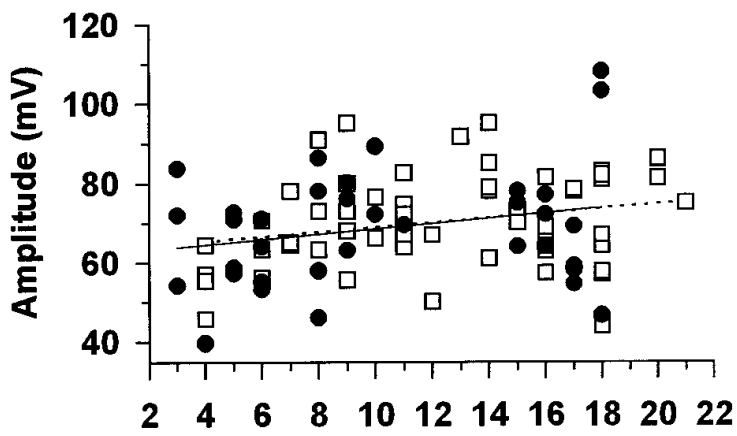

F- Sodium Spikes per Burst

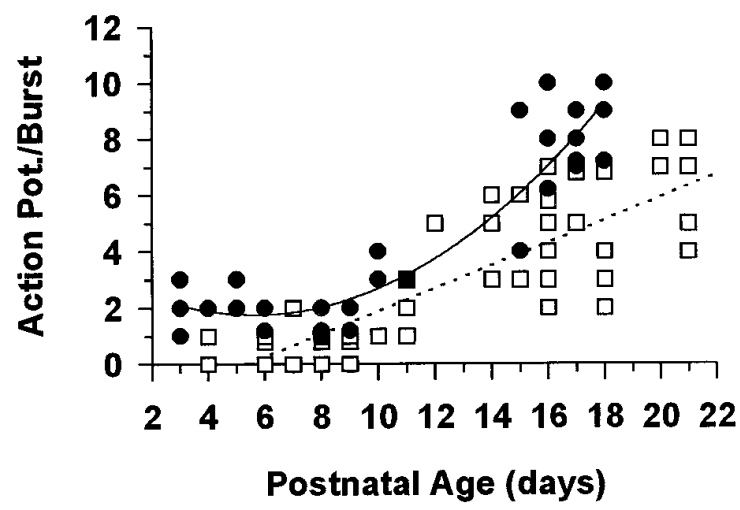

Figure 1. Graphs illustrating the basic membrane properties and the firing characteristics of RTN (solid symbols) and VPN (open symbols) neurons as a function of postnatal age. $A$, RMPs; statistically significant correlations were found between the RMP and postnatal age for both $\mathrm{RTN}\left(r^{2}=0.452, p<\right.$ $0.0001, n=36)$ and VPN $\left(r^{2}=0.146, p=0.021, n=69\right)$ neurons, the RMP becoming more negative with age. RTN neuron values were best fit by a linear regression and VPN neuron values by a second-order polynomial regression. $B$, Membrane time constant $\left(\tau_{\mathrm{m}}\right)$. Statistically significant correlations were found between $\tau_{\mathrm{m}}$ and postnatal age for both RTN $\left(r^{2}=0.394, p<0.0001, n=35\right)$ and VPN $\left(r^{2}=0.283, p<0.0001, n=64\right)$ neurons, $\tau_{\mathrm{m}}$ becoming shorter with age. Both RTN and VPN neuron values were best fit by a linear regression. $C$, Membrane input resistance $\left(R_{\text {in }}\right)$. Statistically significant correlations were found between $R_{\text {in }}$ and postnatal age for both RTN $\left(r^{2}=0.389, p<0.0001, n=37\right)$ and VPN $\left(r^{2}=0.402, p<0.0001, n=66\right)$ neurons, $R_{\text {in }}$ becoming smaller with age. RTN neuron values were best fit by a linear regression and VPN neuron values by a second-order polynomial regression. $D$, The duration of the action potential measured at $50 \%$ of the amplitude. Statistically significant correlations were found between the duration of the action potential and postnatal age for both RTN $\left(r^{2}=0.413, p=0.0001, n=29\right)$ and VPN $\left(r^{2}=0.644, p<0.0001, n=46\right)$ neurons, the action potential becoming shorter with age. RTN neuron values were best fit by a linear regression and VPN neuron values by a second-order polynomial regression. $E$, Amplitude of the action potential. No statistically significant correlation was found between the size of the action potential and postnatal age for RTN $\left(r^{2}=0.055, p=0.173, n=35\right)$ or VPN $\left(r^{2}=0.058, p=0.692, n=58\right)$ neurons. $F$, Maximum number of $\mathrm{Na}^{+} / \mathrm{K}^{+}$action potentials in a burst in response to either depolarizing current with the membrane in a hyperpolarized state or as a rebound from a large hyperpolarizing current pulse with the membrane depolarized. Statistically significant correlations were found between the number of sodium action potentials and postnatal age for both RTN $\left(r^{2}=0.859\right.$, $p<0.0001, n=34)$ and VP $\left(r^{2}=0.678, p<0.0001, n=57\right)$ neurons, the number increasing with age. RTN neuron values were best fit by a second-order polynomial regression and VPN neurons values by a linear regression. In all graphs, each dot represents the value from an individual neuron. The number of neurons was 36 and $69(A), 35$ and $64(B), 37$ and $66(C), 29$ and $46(D), 35$ and $58(E)$, and 34 and $57(F)$ for RTN and VPN, respectively. 


\section{A- RTN P3}

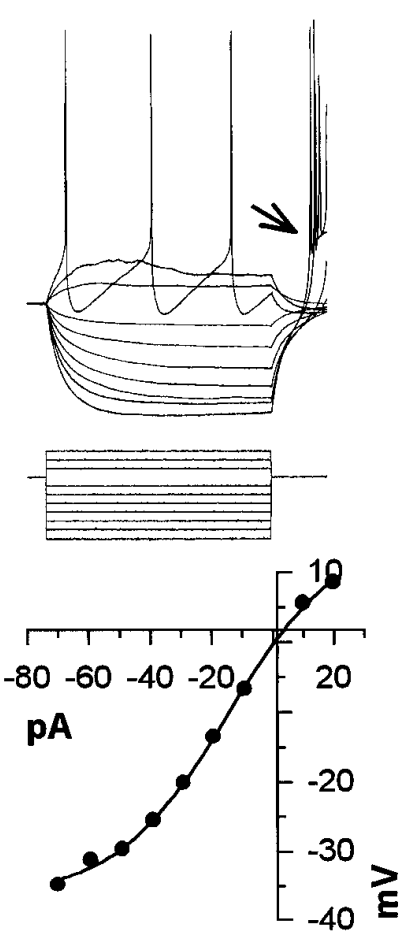

D- VP P4
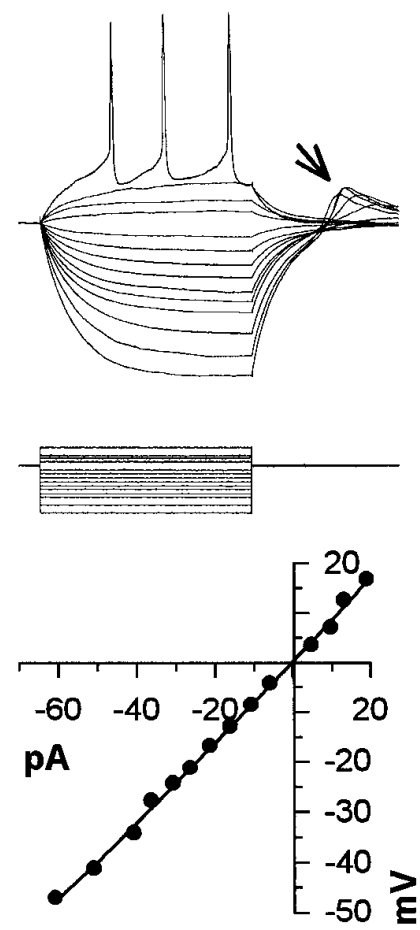

B- RTN P6
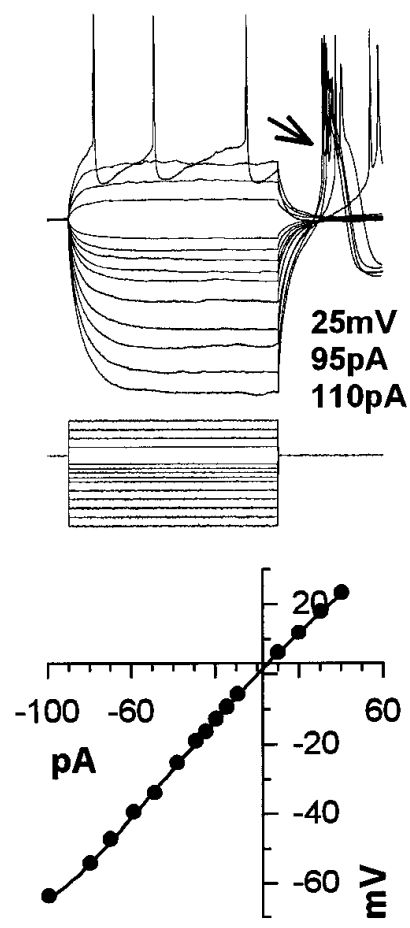

E- VP P8
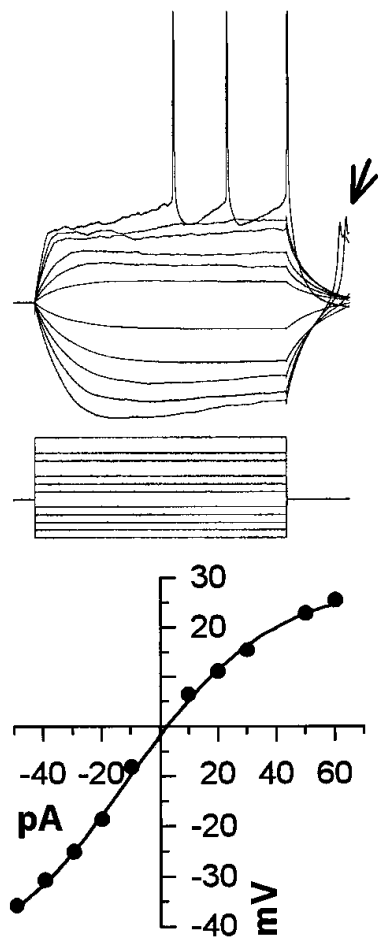

\section{C-RTN P10}

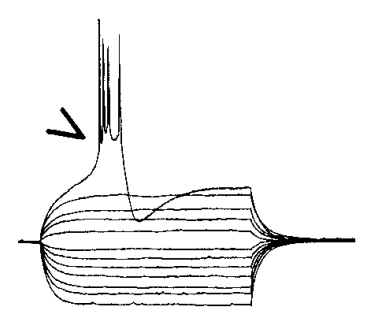

$200 \mathrm{~ms}$
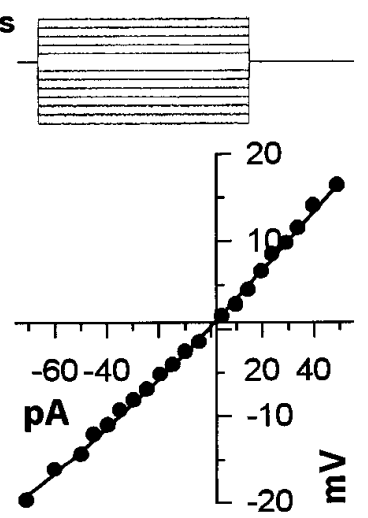

F- VP P11
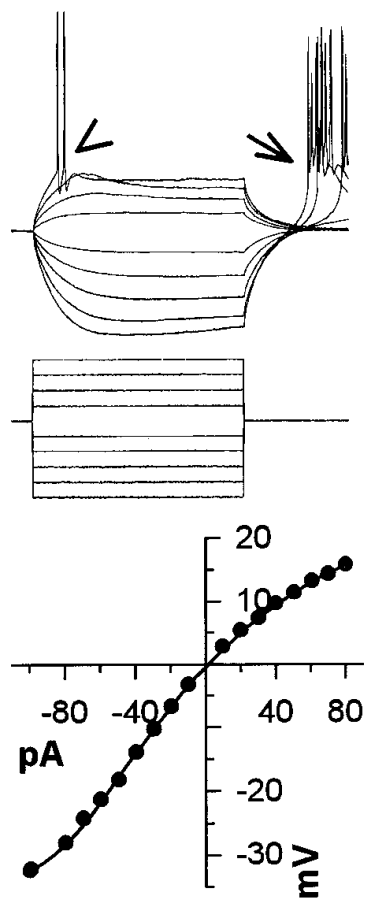

Figure 2. Responses (top row) of RTN $(A-C)$ and VPN neurons $(D-F)$ to depolarizing and hyperpolarizing current pulses (middle row) from RMP and $I-V$ relationships (bottom row) for RTN neurons from P3 $(A), \mathrm{P} 6(B)$, and P10 $(C)$ animals and VPN neurons from P4 $(A)$, P8 $(B)$, and P11 $(C)$ animals. RMP was $-52 \mathrm{mV}$ in $A,-52 \mathrm{mV}$ in $B,-62 \mathrm{mV}$ in $C,-48 \mathrm{mV}$ in $D,-60 \mathrm{mV}$ in $E$, and $-59 \mathrm{mV}$ in $F$.

and the last neuron in which no $\mathrm{Na}^{+} / \mathrm{K}^{+}$spike overrode the LTS was found at P9. Because of the relative absence of $\mathrm{Na}^{+} / \mathrm{K}^{+}$ spikes in VPN neurons before P11, LTS could be observed in relative isolation (e.g., arrows in Fig. $4 D, E$ ), and it was evident that the LTS became much larger with increasing age until, at P11, spiking was regularly observed, the LTS having become sufficiently large presumably to reach spiking threshold (Fig. 4F). After P11 (Fig. $1 F$ ) there was a dramatic increase in the capacity 


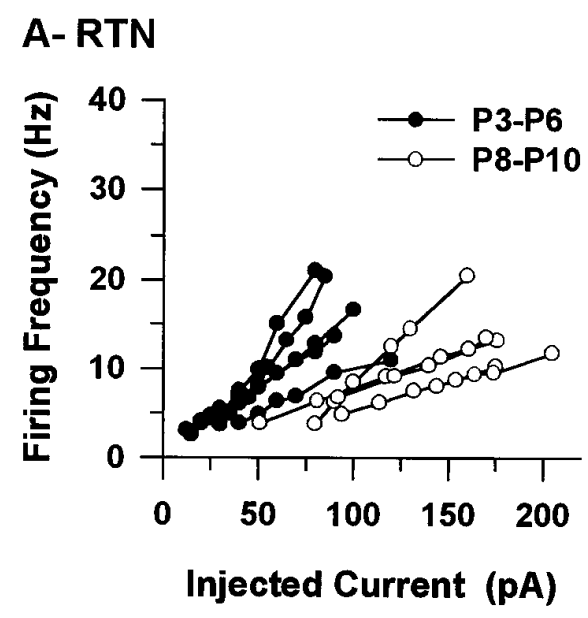

Figure 3. Characteristics of tonic firing in RTN and VPN neurons. $A$ and $B$ show the firing frequency of the first interspike interval as a function of the current injected for RTN $(A)$ and VPN $(B)$ neurons at two different postnatal ages. Measurements were obtained from responses to $500 \mathrm{msec}$ depolarizing current pulses administered from RMP in neurons younger than P8. In older neurons, the membrane was depolarized with DC current before the current pulse to produce pure tonic firing; the DC current was added to the current pulse. $C$ shows the regression lines fitted to the frequency curves from $A$ and $B . D$ shows plots of the slopes of the regression lines from $C$ as a function of postnatal age (correlation coefficients were all $>0.966$ ).

\section{C-Regression Lines}

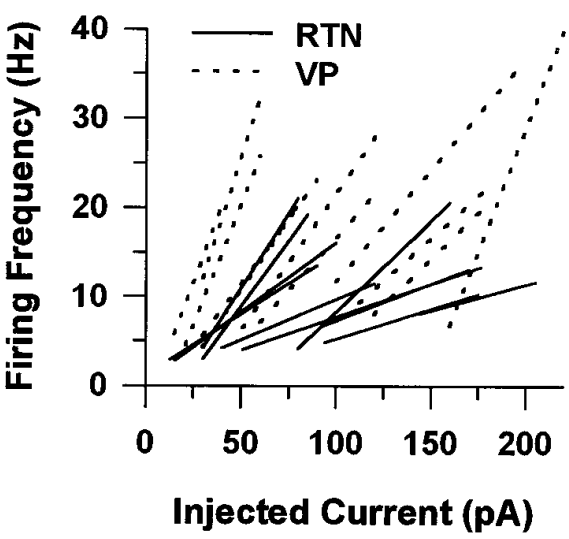

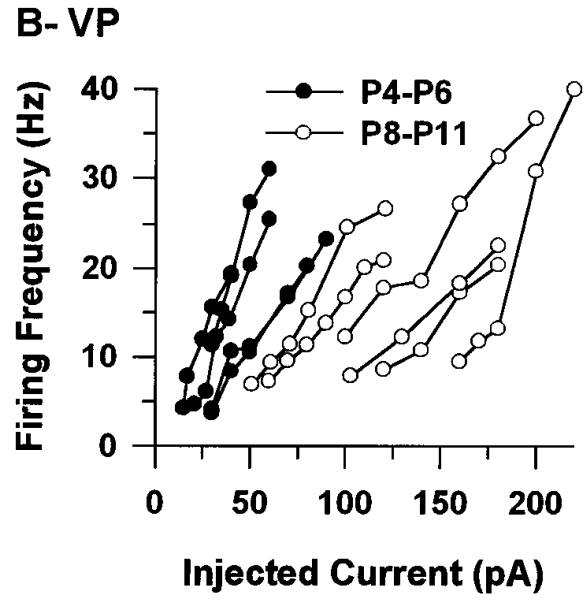

D- Frequency

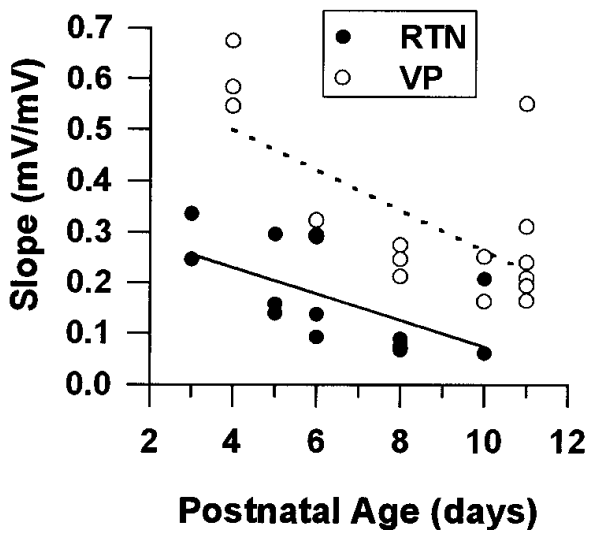

of both RTN and VPN neurons to fire bursts of action potentials, and there was a sudden increase in the number of $\mathrm{Na}^{+} / \mathrm{K}^{+}$action potentials per burst.

In both RTN and VPN neurons younger than P7, LTS could only be elicited from RMP if the neuron was hyperpolarized (Figs. $2 A, B, D, E$, arrows; $4 A, B, D, E)$, and both RTN and VPN neurons normally fired tonically rather than in bursts when depolarized with current pulses from RMP. Starting at P7, it was possible to detect small, low-threshold $\mathrm{Ca}^{2+}$ currents when depolarizing current was applied from RMP, but bursts were not consistently evoked from RMP before P10-P11. By contrast, after P10-P11, both RTN and VP neurons fired in bursts when depolarized from RMP (Figs. 2C,F, arrowheads; 4C,F). In addition, RTN neurons lost the ability to burst on passive membrane repolarization succeeding a hyperpolarizing current pulse, whereas VPN neurons retained the capacity to produce a rebound burst after a pulse of hyperpolarizing current from RMP (Fig. $2 F$, arrow).

These findings show that the RMP and the activation potential of RTN and VPN neurons change relative to one another during postnatal development. The RMP of RTN neurons becomes negative with respect to the activation threshold of the LTS with age, whereas that of VPN neurons lies within the activationinactivation range of the LTS. RTN neurons, thus, will fire rebound bursts only if their membrane is depolarized in the activation range of the LTS before the administration of a hyperpolarizing pulse (data not shown) (see Warren et al., 1994). Measurements of the activation potential of the LTS showed that it remained constant throughout the period studied in both RTN and VPN neurons (Fig. 4G), (c.f., Pirchio et al., 1990). At all ages, the activation potential of the LTS of RTN neurons was on average $>15 \mathrm{mV}$ depolarized compared with that of VPN neurons, with averages of $-54.8 \pm 1.1 \mathrm{mV}$ and $-70.1 \pm 0.8 \mathrm{mV}$ for RTN and VPN neurons, respectively. That difference between RTN and VPN neurons remained constant during the period studied, suggesting an early differential expression of the LTS and implying that intrinsic membrane properties typical of the adult cells are present very early during postnatal development.

When the RMP was compared with the activation potential of the LTS (Fig. 4H), it was found to be positive to the LTS activation potential in the youngest RTN neurons studied and became negative to it at the end of the first postnatal week as the RMP became more negative. At this age, the LTS could be activated either by a direct depolarization or as a rebound from a hyperpolarization. As the RMP became more negative, the LTS could only be activated at rest by a depolarization. By contrast, at all ages, the RMP of VPN neurons was above the activation potential of the LTS, consistent with the fact that the LTS was activated at the end of a hyperpolarizing pulse applied from the RMP. Accurate measurement of the inactivation potential of the LTS was not possible without blocking $\mathrm{Na}^{+} / \mathrm{K}^{+}$spikes in RTN neurons because of the overlap in the tonic firing threshold and because the action potential threshold was within the activation range of the LTS.

In summary, before P12, VPN and RTN neurons are already physiologically distinct. Although the physiological properties typical of each class of neuron are present at P3, they remain 

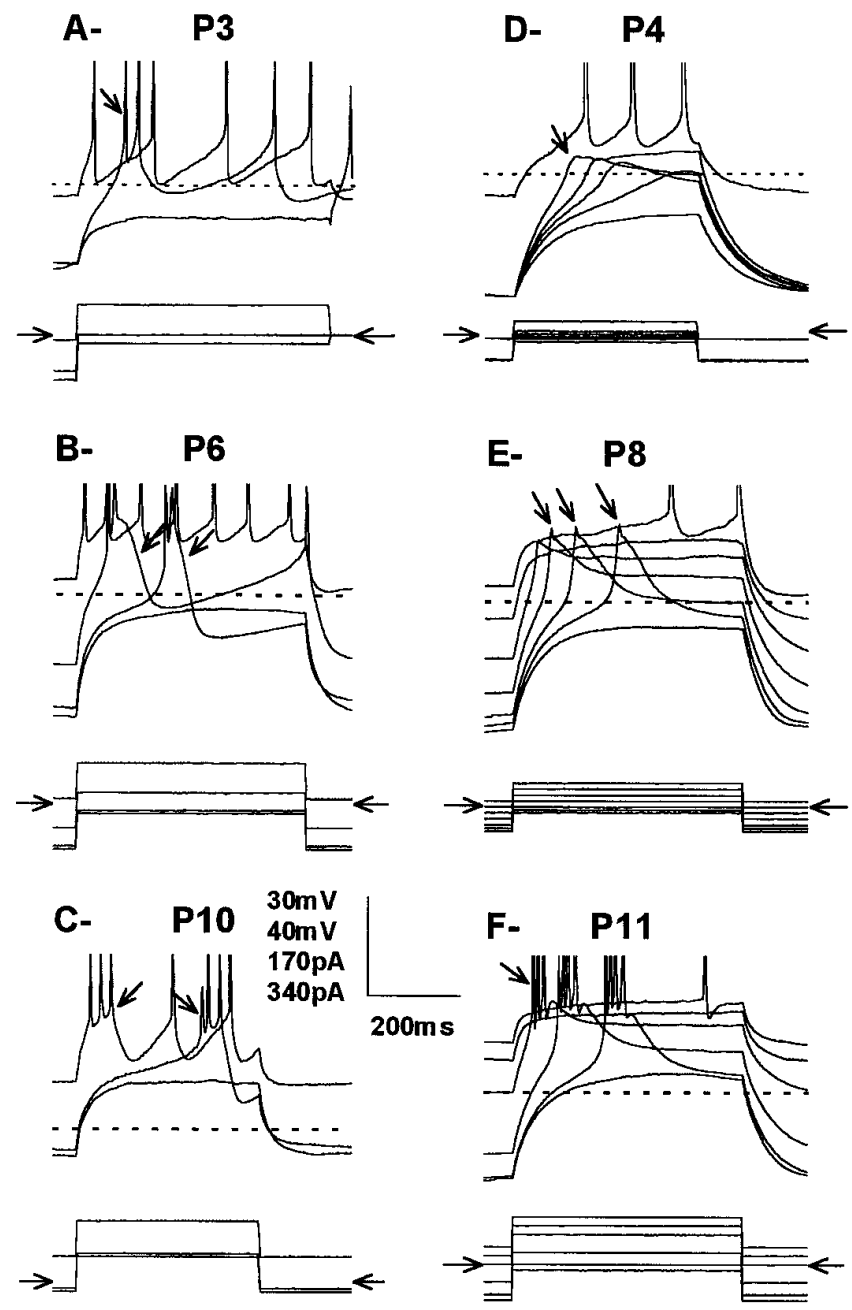

\section{G- LTS Activation Potential}

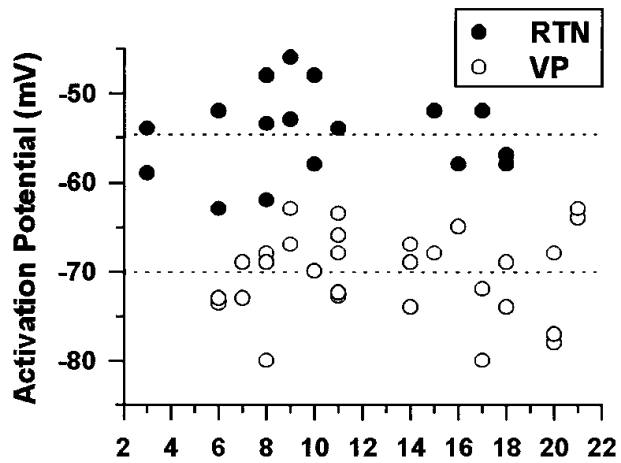

\section{H- $\triangle$ RMP - LTS Activation Potential

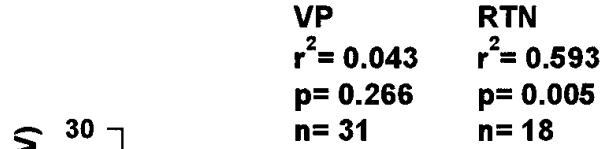

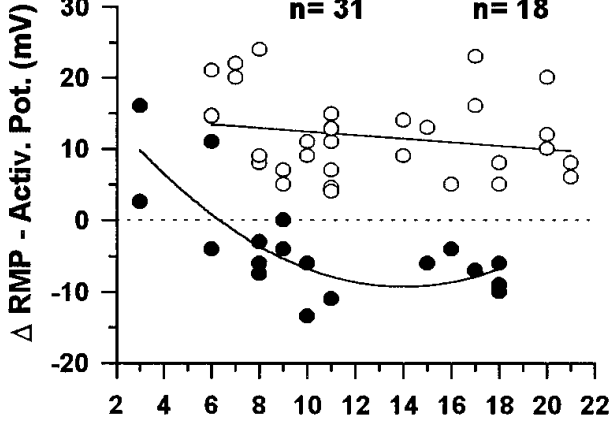

Postnatal Age (days)

Figure 4. Responses of the RTN neurons $(A-C)$ from Figure 2, $A-C$, and of the VPN neurons $(E, F)$ from Figure 2, $D-F$, to current pulses generating LTSs. Top row of each panel shows the voltage response, the dotted line corresponds to the RMP, and the arrows indicate examples of bursts of $\mathrm{Na}^{+} / \mathrm{K}^{+}$ spikes or $\mathrm{Ca}^{2+}$ depolarizations. An evoked LTS can be seen in all traces except the bottom of each panel. Bottom row of each panel shows the current pulses, and the horizontal arrows indicate the level of no current injection. In $A$, the constant depolarizing pulses were given after a 500 msec hyperpolarizing pulse of varying amplitude, and both pulses were turned off simultaneously. In $B-F$, the neuron received a constant depolarizing current pulse while held at different hyperpolarized and depolarized membrane potentials, with a 450 or 750 msec pulse of current before and 450 or 250 msec after. Vertical scale bar: $A, C-F, 30 \mathrm{mV} ; B, 40 \mathrm{mV} ; A-D, 170 \mathrm{pA} ; E, F, 340 \mathrm{pA}$. The $\mathrm{Na}^{+} / \mathrm{K}^{+}$spikes are truncated in $A-F$. $G$, Activation potential of the LTS in RTN $(n=18)$ and VPN $(n=31)$ neurons as a function of postnatal age measured in current clamp by giving large $500 \mathrm{msec}$ depolarizing current pulses 500-700 $\mathrm{msec}$ after the beginning of a $1400 \mathrm{msec}$ hyperpolarizing current pulse that was incremented with $-5 \mathrm{pA}$ steps; the depolarizing pulses were sufficient to produce a depolarization of at least $30 \mathrm{mV}$. The activation potential was determined as the highest potential at which LTS could not be detected at the crest of the depolarizing pulse. The same procedure was used for RTN and VPN neurons. No statistically significant correlations were found between the LTS activation threshold and postnatal age for either RTN $\left(r^{2}=0.003, p=0.838, n=18\right)$ or VPN $\left(r^{2}<0.001, p=0.999, n=31\right)$, but statistically significant difference was found between RTN and VPN $(t s=-10.958, \mathrm{df}=47, p<0.0001)$. The horizontal dotted lines indicate the mean for RTN (top, $-54.8 \pm 1.1 \mathrm{mV}$ ) and VPN (bottom, $-70.1 \pm 0.8 \mathrm{mV}$ ) neurons. $H$, The difference between the RMP and the LTS activation potential (as measured in $G$ ) as a function of postnatal age. The dotted line at zero indicates the LTS activation potential. Circles above and below the LTS activation potential line indicate neurons with their RMP above and below LTS activation threshold. The solid lines are the regression lines. A statistically significant correlation between the activation potential and postnatal age was found for RTN neurons but not for VPN neurons. RMP was $-52 \mathrm{mV}$ in $A,-52 \mathrm{mV}$ in $B,-62 \mathrm{mV}$ in $C,-48 \mathrm{mV}$ in $D,-60 \mathrm{mV}$ in $E$, and $-59 \mathrm{mV}$ in $F$.

immature up to $\mathrm{P} 10$. This immaturity is characterized by a relatively depolarized membrane, slower action potentials, and a weakness in the ability of the LTS to generate $\mathrm{Na}^{+} / \mathrm{K}^{+}$spikes, especially in VPN neurons. By P12, they acquire all the characteristics of the adult neurons.

\section{Synaptic responses in RTN}

Postsynaptic responses could be evoked in RTN neurons as early as $\mathrm{P} 3$ by electrical stimulation of the internal capsule (Fig. $5 A$ ). These responses were depolarizing at RMP and most likely were monosynaptic EPSPs evoked by the activation of collaterals of corticothalamic and/or thalamocortical fibers in the RTN (Warren et al., 1994). The amplitude of the EPSPs increased with increase in stimulus intensity, and in all RTN neurons tested, the depolarization became sufficiently large to reach firing threshold (Fig. 5).

From P3 to P7, subthreshold synaptic responses in RTN neurons had a relatively slow rising phase and appeared as a single continuous EPSP. With stimuli just above threshold, the rising 
Figure 5. Responses of RTN neurons to electrical stimulation of the internal capsule as a function of age $(A-C)$ and the pharmacology of the synaptic response $(D-G)$. $A$, Five superimposed traces of the synaptic responses in a RTN neuron recorded in a slice from a P3 mouse using stimulus intensities of 8-30 V. Arrowhead indicates a slow depolarization after a synaptically evoked action potential. $B$, Six superimposed synaptic responses in a RTN neuron from a P8 slice using stimulus intensities of $1.5-15 \mathrm{~V}$. The arrows indicate a slow depolarization that followed a faster depolarization and led the neuron to firing threshold. The arrowheads indicate a slow depolarization that followed evoked action potentials. $C$, Three superimposed synaptic responses of a RTN recorded in a P10 slice using stimulus intensities of 5-15 V. The arrow indicates a slow depolarization that eventually lead the neuron to fire a burst of action potentials. The arrowhead indicates a slow decaying depolarization with subthreshold stimulus, and the double arrowheads indicate the delayed depolarization after the burst with high-intensity stimulus. $D$, Effects of excitatory amino acid receptor antagonists APV (50 $\mu \mathrm{M})$ and CNQX $(20 \mu \mathrm{M})$ on the neuron shown in $C$, with a stimulus of the internal capsule of $12 \mathrm{~V}$. The two nearly overlapping traces represent the control and the recovery, respectively, and the arrow points to the response recorded $13 \mathrm{~min}$ after adding the antagonists to the perfusing medium. $E$, Effects of excitatory amino acid receptor antagonist APV $(50 \mu \mathrm{M})$ on a P10 neuron. The stimulus of the internal capsule was $12 \mathrm{~V}$. The two nearly overlapping traces are the control and the recovery, and the arrow points to the response recorded $8 \mathrm{~min}$ after adding APV to the perfusing medium. The arrowhead indicates the after burst hyperpolarization that was slightly larger with APV. $F$, Effects of the non-NMDA receptor antagonist CNQX $(20 \mu \mathrm{M})$ on a neuron in a P8 slice. The arrow indicates a slow EPSP that remains 20 min after the addition of CNQX to the superfus-

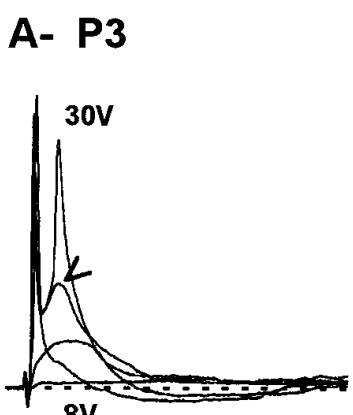

$8 \mathrm{~V}$

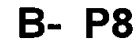

C- P10
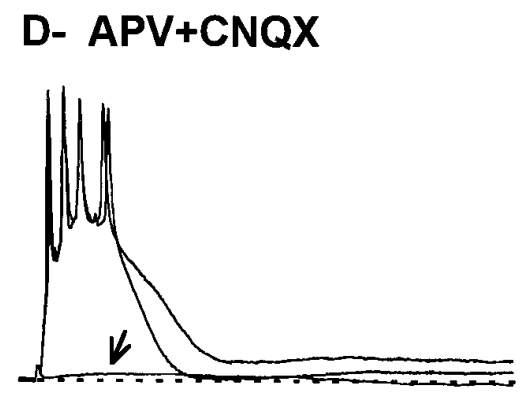

F- CNQX

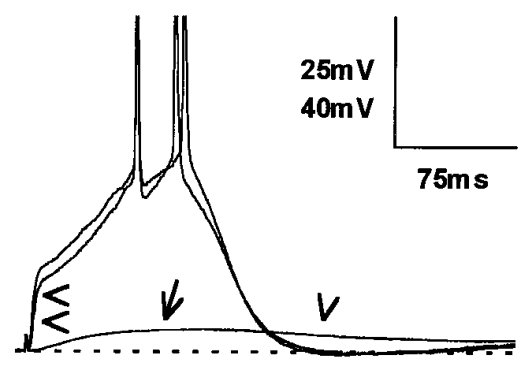

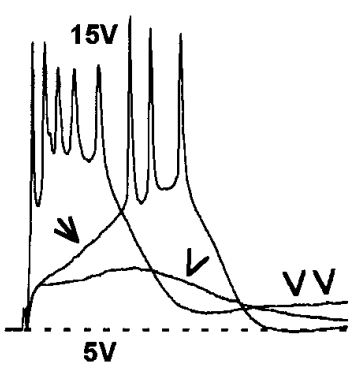

E- APV

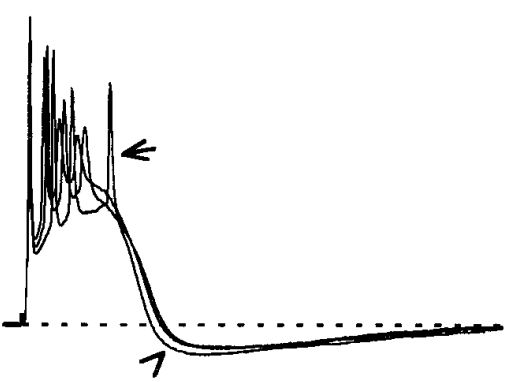

G- BMI

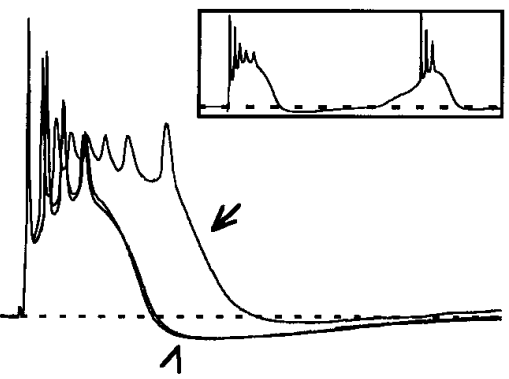
ing medium. The arrowhead indicates the region

of overlap of the slow EPSP and the afterhyperpolarization seen in the absence of CNQX. The double arrowhead indicates the early EPSP, which is blocked by CNQX. $G$, Effects of the $\mathrm{GABA}_{\mathrm{A}}$ receptor antagonist BMI $(10 \mu \mathrm{M})$ on the neuron shown in $E$, with a $12 \mathrm{~V}$ stimulus to the internal capsule. The two nearly overlapping traces represent the control and the recovery; the arrow points to the response recorded 10 min after adding the antagonist to the perfusing medium. The arrowhead indicates the afterburst hyperpolarization that was much larger during control and recovery than with BMI in the bath. The inset shows a response of the same neuron to the same stimulus but only $30 \mathrm{sec}$ after the perfusing solution was switched from control to BMI; the frame represents $550 \mathrm{msec}$ by $95 \mathrm{mV}$. The RMP was $-50 \mathrm{mV}$ in $A,-65 \mathrm{mV}$ in $B$ and $F,-62 \mathrm{mV}$ in $C$ and $D$, and $-64 \mathrm{mV}$ in $E$ and $F$. Vertical scale bars: $A, C, D, 25 \mathrm{mV} ; B, E, F, 40 \mathrm{mV} . \mathrm{Na}^{+} / \mathrm{K}^{+}$action potentials in $F$ are truncated.

phase of the EPSP was faster and in continuity with the rising phase of the single action potential. By increasing the stimulus, a depolarizing potential emerged during the repolarizing phase of the initial action potential (Fig. 5A, arrowhead), and from this depolarization a second action potential could be evoked by further increasing the stimulus. Thus, the typical synaptic responses recorded during the first postnatal week consisted of an initial action potential evoked at short latency, followed by additional action potentials emerging from a detectable secondary depolarization.

At the beginning of the second week, two components could be detected in the subthreshold synaptic depolarizations: an initial fast component that was followed by a second, slowly decaying component (Fig. 5B, single arrowhead in $C$ ). A slight increase in stimulus strength that had little effect on the initial fast depolarization resulted in an increase in the slow, delayed depolarizing component; after several milliseconds, this reached spiking threshold, and either a single action potential (Fig. 5B, arrows) or an all-or-none burst was discharged (Fig. $5 C$, arrow). Character- istically, in RTN neurons from $\mathrm{P} 7$ to $\mathrm{P} 9$, a single action potential was evoked at threshold, whereas at P10 and in older RTN neurons to $\mathrm{P} 21$, a burst of action potentials was evoked at threshold (Fig. $6 A$ ). In cases in which there was only a single $\mathrm{Na}^{+} / \mathrm{K}^{+}$ action potential evoked around threshold, this action potential was also followed by a depolarizing hump that slowly decayed (Fig. 5B, arrowheads). With stronger stimuli, the initial fast depolarizing component led readily to the firing of a burst of action potentials (Fig. $5 B, C$ ), and in most neurons, a burst of action potentials could be generated (Fig. $6 A$ ). The higher number of action potentials synaptically evoked in RTN neurons older than P12 was not apparently attributable to an increase in synaptic strength, because the stimulus threshold was the same in slices from animals younger than P12 as it was in slices from older animals $(5.4 \pm 1.2$ and $4.4 \pm 0.4 \mathrm{~V}$, respectively; $t s=0.267$, $\mathrm{df}=$ $18, p=0.792)$. More likely, it was attributable to the activation of the LTS by the synaptic depolarization in a manner similar to that observed with current depolarization (see above).

The latency to the first synaptically evoked potential depended 


\section{A- $\mathrm{Na}^{+}$Action Potentials}

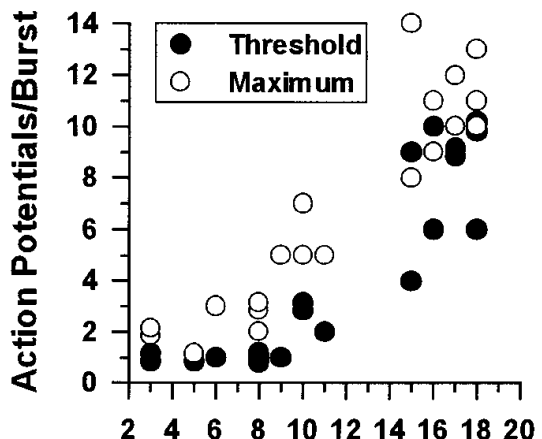

\section{B- Latency}

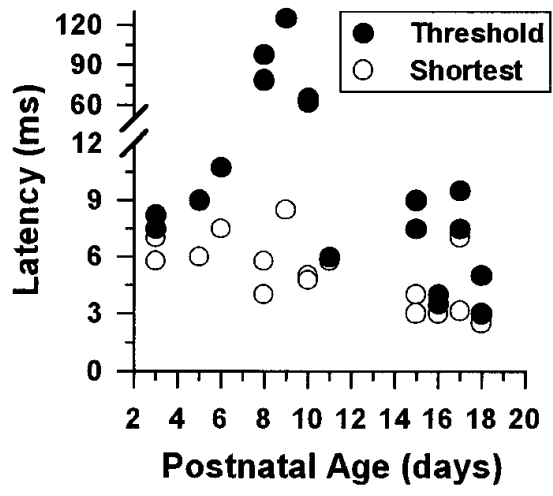

Figure 6. Synaptic responses of RTN neurons. $A$, Number of $\mathrm{Na}^{+} / \mathrm{K}^{+}$ action potentials evoked at threshold (solid circles) and the maximum number that could be evoked (open circles) in RTN neurons by electrical stimulation of the internal capsule as a function of age. Statistically significant differences were found between neurons from animals younger than P12 and those older than P12 for both the number of spikes evoked at threshold $\left(H_{[1]}=15.2, p<0.0001\right)$ and the maximum number of spikes that could be evoked $\left(F_{[1,18]}=79.9, p<0.0001\right)$. Each circle represents the value from a single neuron, because in some cases, the number of spikes has been changed by \pm 0.2 on the plot to make all circles visible. $B$, Latency between stimulus onset and the peak of the first evoked action potential at stimulus threshold (solid circles) and the shortest latency recorded (open circles) in the same set of neurons. The number of neurons was 21 in $A$ and 17 in $B$.

on stimulus strength. With strong stimuli, it decreased with postnatal age (Fig. 6B, open circles), whereas close to threshold, the latency was stable for most of the period studied with the exception of P8-P10, during which the latency of the first action potential increased severalfold (Figs. $5 B, C ; 6 B$, solid circles). This delay was apparently attributable to the slow activation of the LTS on which the action potential was generated. In older slices, the latency was much less affected by the stimulus strength.

The addition of pharmacological agents to the perfusing medium provided clues about the neurotransmitter involved in the synaptic response of RTN neurons. When both APV and CNQX were simultaneously added to the perfusing medium at all ages, the response of RTN neurons to stimulation of the internal capsule was virtually abolished, indicating that the response was mediated by glutamate receptors (Fig. $5 D$, arrow). The addition of each drug separately only partially abolished the response, sug- gesting that both NMDA and non-NMDA receptors were involved (Fig. $5 E, F$ ). APV alone produced only a small reduction in the depolarization underlying the synaptically driven burst; this resulted in a decrease in firing frequency during the burst without a reduction in the number of $\mathrm{Na}^{+} / \mathrm{K}^{+}$action potentials (Fig. $5 E$ ). It also resulted in a slightly larger afterhyperpolarization (Fig. $5 E$, arrowhead). Similar findings were observed in RTN neurons from animals at all ages from $\mathrm{P} 3$ to $\mathrm{P} 18(n=7)$. CNQX alone produced much more dramatic effects (Fig. $5 F$ ). The initial fast depolarization (Fig. $5 F$, double arrowhead) was completely abolished, showing that it was mediated by non-NMDA receptors. Only a small, slow depolarization, presumably mediated by NMDA receptors, remained. This slow EPSP overlapped the afterhyperpolarization observed in the control condition (Fig. $5 F$, single arrowhead). Its abolition when APV was added to the medium (e.g., Fig. 5E) probably explained the decrease in the depolarization and the apparent increase in the afterhyperpolarization.

Because RTN neurons are GABAergic and synaptically interconnected (Houser et al., 1980; Deschênes et al., 1985; Yen et al., 1985), the effects of the $\mathrm{GABA}_{\mathrm{A}}$ receptor antagonist BMI on the response of RTN neurons were examined $(n=7)$. The most striking effect of BMI at any age was in prolonging the duration of the synaptically driven burst response and, hence, the number of action potentials discharged per burst (Fig. 5F) (see also Bal and McCormick, 1995a). With BMI in the perfusing medium, the afterhyperpolarization was also reduced significantly as compared with controls (Fig. 5G, arrowhead). This suggests that GABAergic inhibition acts to shunt the burst of action potentials in RTN cells. This GABAergic influence probably comes from neighboring RTN neurons, because none of the known sources of GABAergic projections to the RTN reach it by way of the internal capsule. In the P10 neuron shown in Figure $5 F$, a recurring burst generated by a typical barrage of EPSPs (see Warren et al., 1994) was also noted during the early wash-in and late wash-out of BMI, but this was not present when BMI effects were maximal or during the control period (Fig. 5F, inset). Presumably, BMI acted faster on increasing RTN neuron bursts than in blocking the IPSPs generated in VPN neurons by the RTN burst discharges, thus producing a larger IPSP in the VPN neurons that was sufficient to produce a rebound excitation feeding forward onto the RTN neurons to reinforce their bursting. We did not observe oscillations in RTN neurons younger than P11, except in the presence of the slight reduction in $\mathrm{GABA}_{\mathrm{A}}$ receptor inhibition produced early in the application of BMI. At P12 and older ages, single-pulse stimulation of the internal capsule invariably caused most RTN and VPN neurons to oscillate at $2-4 \mathrm{~Hz}$ for periods of $8 \mathrm{sec}$ or more (Warren et al., 1994)

The present results show that RTN neurons receive functional glutamatergic innervation as early as P3 and that this excitatory input can be sufficient to induce RTN neurons to fire and likely exert an inhibitory influence on VPN neurons (see following section), but this inhibition is apparently insufficient to produce rebound bursting and, therefore, oscillations in the network.

\section{Synaptic responses in VPN}

Electrical stimulation of the internal capsule also evoked synaptic responses in VPN neurons at all ages studied (Fig. $7 A-C$ ). The synaptic response of VPN neurons was usually dominated by a large hyperpolarization at RMP that reversed at potentials negative to the RMP. This response is considered to be an inhibitory postsynaptic potential mediated by GABAergic receptors (Fig. 8) and generated by the activation of RTN neurons (Warren et al., 


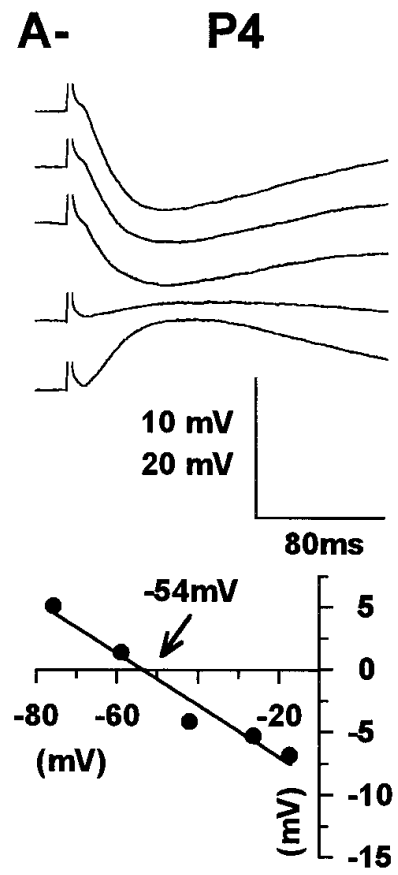

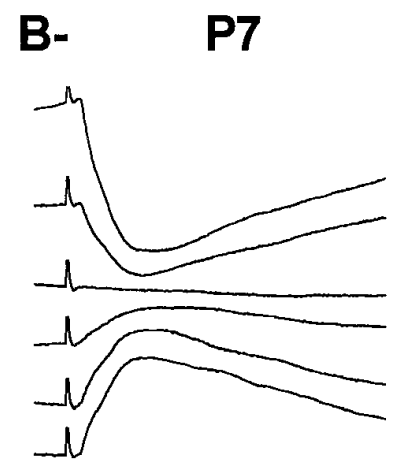

C- $\quad$ P10
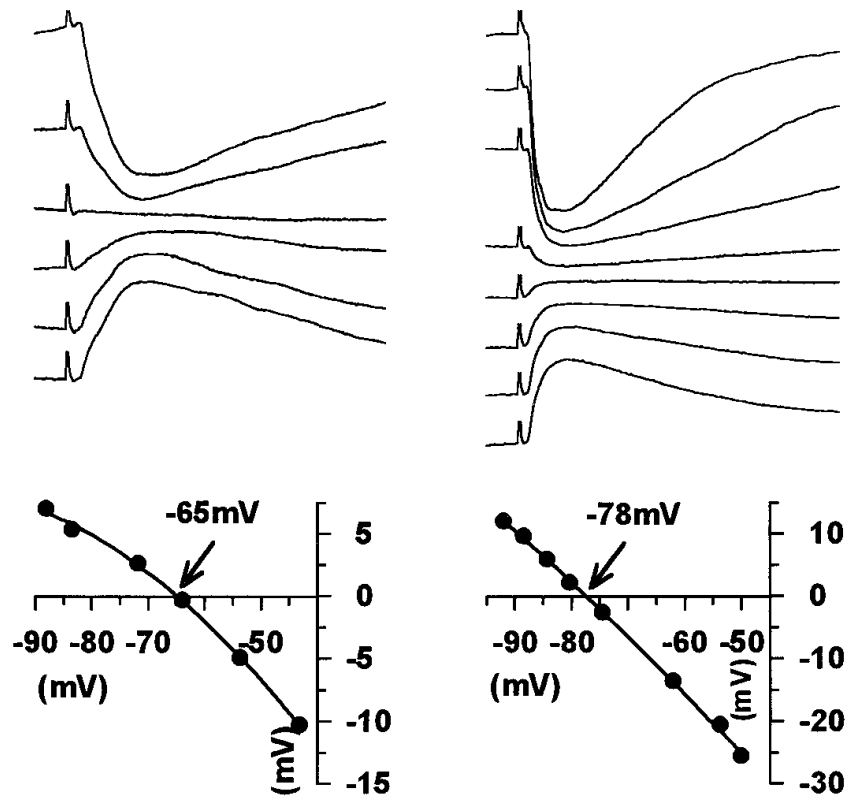

Figure 7. $A-C$, Current-clamp recording of synaptic responses evoked in VPN neurons by electrical stimulation of the internal capsule in slices from $\mathrm{P} 4(A), \mathrm{P} 7,(B)$, and P10 $(C)$ animals and their corresponding $V_{\mathrm{R}}-V_{\mathrm{m}}$ curves obtained close to the peak of the response. The voltage traces have been aligned on the portion between the stimulus artifact and the beginning of the response. The more positive responses were obtained from the more negative membrane potentials and vice versa, as shown in the $V_{\mathrm{R}}-V_{\mathrm{m}}$ plots. Each trace represents the average of four responses. The arrows and the $\mathrm{mV}$ values on the $V_{\mathrm{R}}-V_{\mathrm{m}}$ curves indicate the reversal potential of the responses. RMP was -50 $\mathrm{mV}$ in $A$ and $-59 \mathrm{mV}$ in $B$ and $C$. The vertical bar represents $10 \mathrm{mV}$ for $A$ and $B$ and $20 \mathrm{mV}$ for $C . D$, Graph showing the reversal potential of the IPSP and/or IPSC recorded in VPN neurons as a function of postnatal age. The total number of neurons was 42 (18 younger than P12 and 24 P12 and older). Data were fit with a thirdorder polynomial regression.

\section{D- Reversal Potential of IPSP/IPSC}

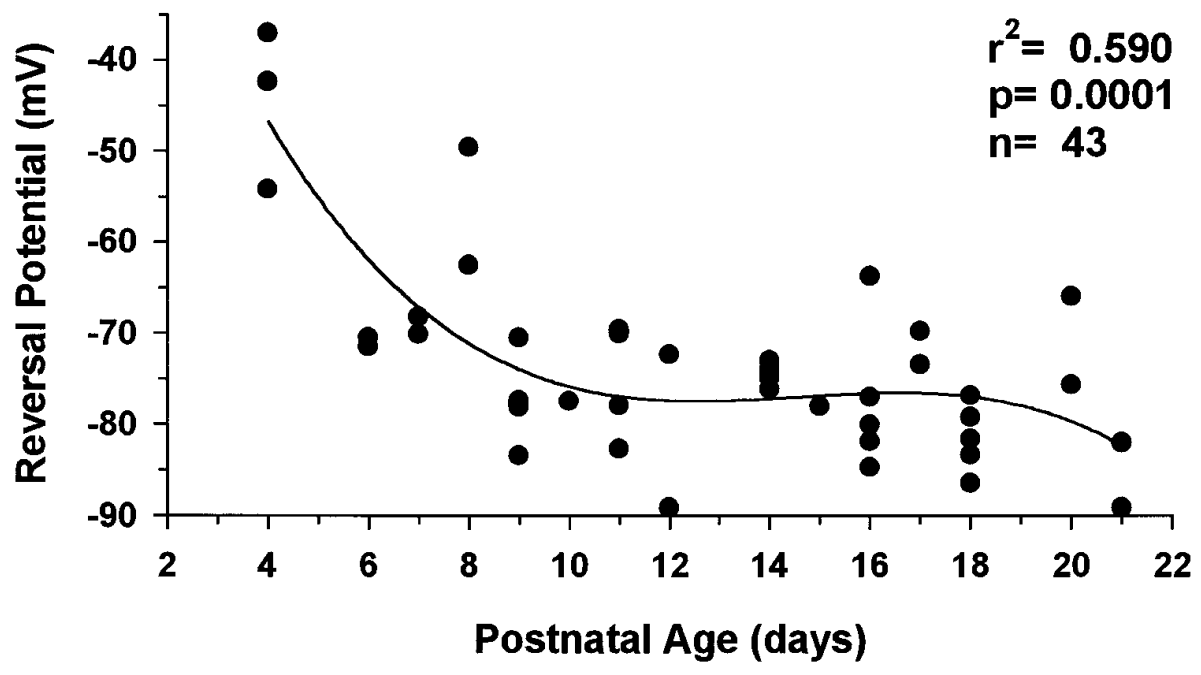

1994). In some younger neurons, the response was depolarizing at RMP and the reversal potential above the RMP; nevertheless, it was abolished when BMI was added to the perfusing medium, showing that it was also mediated by GABA.

The inhibitory VPN response was monophasic at all ages and always displayed a quasilinear function in relation to the membrane potential at the peak of the response. The IPSP became shorter at depolarized and, to a lesser degree, at hyperpolarized membrane potential, especially in older neurons (e.g., Fig. 7C). This was presumably attributable to a decrease in the apparent $R_{\text {in }}$ (as indicated by the shape of the $I-V$ curves, Fig. $2 D-F$ ) and a substantial shortening of the $\tau \mathrm{m}$ as the membrane potential was moved away from resting. The reversal potential became more negative with age, stabilizing slightly below $-75 \mathrm{mV}$ at $\sim \mathrm{P} 12$ (Fig. $7 D$ ). This large inhibitory response was sometimes preceded by a small depolarization considered to be an EPSP produced by activation of corticothalamic fibers. The frequency of observation of this EPSP increased with age. It was found in only 5/14 neurons at P4-P9 but in 4/5 neurons at P10-P11 (see below).

Although in current clamp recordings, the large hyperpolarizing response appeared smooth, in voltage clamp, the response often appeared as multiple events with variable latencies after the stimulus, particularly in VPN neurons at P3-P7 (Fig. 8A,B). The multiple currents all reversed at a single membrane potential and were completely and reversibly blocked by BMI $(n=7)$, suggesting that they were $\mathrm{Cl}^{-}$currents mediated by $\mathrm{GABA}_{\mathrm{A}}$ receptors. Most likely, they resulted from the asynchronous firing of RTN neurons, because the multiple events tended to fuse together as a stimulus was increased. This phenomenon was not observed in animals older than P10 (data not shown). In many neurons at P3-P7 (Fig. 8A,B), the response became totally flat with BMI in the bath and at any membrane potential, suggesting that the 
A- P4

Control

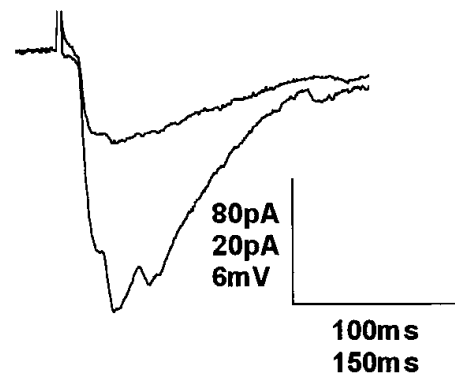

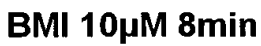

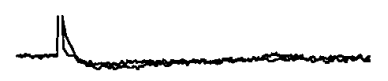

Wash $16 \mathrm{~min}$

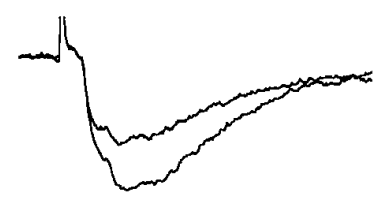

B- P7

Control

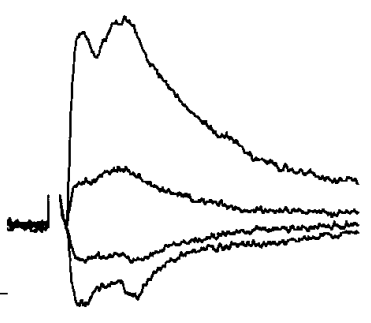

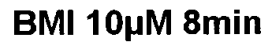

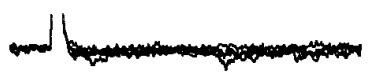

Wash $19 \mathrm{~min}$

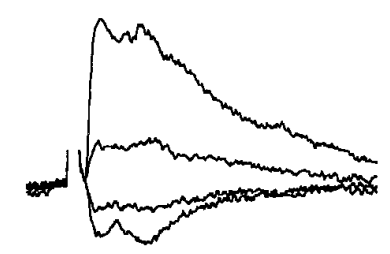

C- P10

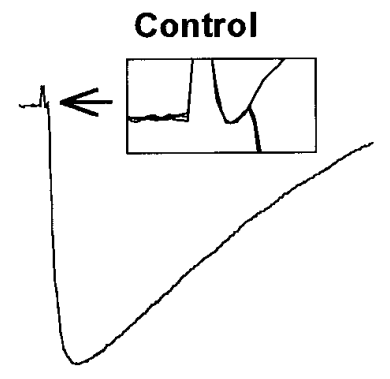

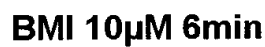

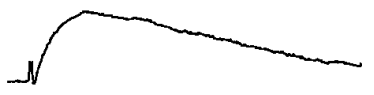

Wash

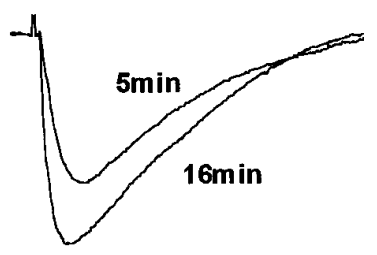

Figure 8. Synaptic responses of the same VPN neurons shown in Figure 7 recorded before (Control), during (BMI $10 \mu \mathrm{M}$ ), and after (Wash) the addition of $10 \mu \mathrm{M}$ bicuculline methiodide to the perfusing medium. $A$, Voltage-clamp recordings at -57 and $-97 \mathrm{mV}$. $B$, Voltage-clamp recordings at $-39,-59,-79$, and $-99 \mathrm{mV}$. $C$, Current-clamp recordings at RMP, $-63 \mathrm{mV}$. The inset shows an enlargement of the peristimulus control, BMI, and recovery period to show that the EPSP that remains in the presence of BMI is actually present during control and recovery but that it is quickly overpowered by the large IPSP. Vertical scale bar: $A, 80 \mathrm{pA} ; B, 20$ $\mathrm{pA} ; C, 6 \mathrm{mV}$. Horizontal scale bar: $A, B$, $100 \mathrm{msec}$;, $150 \mathrm{msec}$.
$\mathrm{GABA}_{\mathrm{A}}$ IPSP/IPSC was the only synaptic response evoked in VPN neurons. In older neurons, it became evident that the IPSP encompassed a small EPSP that was uncovered by BMI (Fig. 8C). The IPSP/IPSC was also completely abolished when CNQX and APV were added to the perfusing medium $(n=4)$, strongly arguing for an inhibitory effect resulting from activation of RTN neurons.

Consistent with what was observed in RTN neurons, the IPSP in VPN neurons could be delayed by varying the intensity of the stimulus at certain postnatal ages. Figure 9 shows $\operatorname{RTN}(A)$ and VPN $(B)$ neurons recorded in the same slice while stimulating at a single location in the internal capsule. The delayed firing of the RTN neuron was matched by the delay in the IPSP recorded in the VPN neuron when the stimulus intensity was varied.

\section{Morphology of RTN and VPN neurons}

\section{Qualitative observations}

At P3, RTN cells and VPN cells are obviously immature (Fig. $10 A, B)$. The somata are smaller than comparable cells at $\mathrm{P} 21$; dendritic fields are also small but composed of a seemingly excessive number of branches, many of which recurve toward the soma instead of extending symmetrically from it as in older animals (e.g., Figs. 11, 12). At P3, the dendrites of both RTN and VPN cells arise from relatively thick primary trunks that do not have a sharp point of origin from the soma, appearing more as extruded continuations of the soma; the subsequent second-order branches, of which three or more can be given off by each primary trunk, are unusually thin and beaded, an appearance not found at older ages. Tertiary branches are uncommon at P3. Some of the dendrites, both large and small, possess small protrusions from which filopodia arise. These are interpreted as growth cones. The dendritic field tends to be radially symmetrical in both RTN and VPN cells at P3. The outline of the soma is less discrete than at later ages because of the multiple (usually 4-6), relatively large dendrites that arise from it.

At P7, RTN cells have acquired a shape not unlike that of the adult cells, whereas VPN cells remain in a state not greatly different from that seen at P3 (Fig. 10C-F). Many of the RTN cells have now taken on the elongated or flattened form of the adult cells. The outline of the soma is more discrete, because the points of origin of the proximal dendrites have become more clearly defined. Dendrites are longer, and the size difference between primary and secondary branches is less great, although the secondary dendrites remain beaded. More tertiary branches and fewer growth cones are seen at this age. The VPN cells at P7 show some increase in the size of their somata and some lengthening of the proximal dendrites, but secondary dendrites, despite their numbers, are still thin, beaded, and unusually short. The diameter of the field $(75-100 \mu \mathrm{m})$ is not greatly different from that at P3.

At P10, RTN cells closely resemble those of the adult, primarily in the configuration of the dendritic field, which is elongated and bitufted on many cells but radially symmetrical in others, even in adjacent cells (Figs. 10G,11). The size differences between primary, secondary, and tertiary dendrites show even less contrast and are typical of adult cells. The number of secondary dendrites is visibly reduced; the tertiary dendrites have become relatively longer than at P7. The dendrites of P10 RTN cells, unlike at the younger ages or in adult cells, have become studded with moderate to high numbers of dendritic spines (Fig. 11). P10 VPN cells are to all appearances unchanged from their state at P7 (Figs. $10 F, 11)$. They have thinner dendrites than RTN cells and few or no spines (Fig. 11, insets).

At P14, RTN cells are somewhat larger than at P10, and their dendritic field structure is adult-like. Most of the cells in the part 


\section{A- RTN Synaptic Bursts}

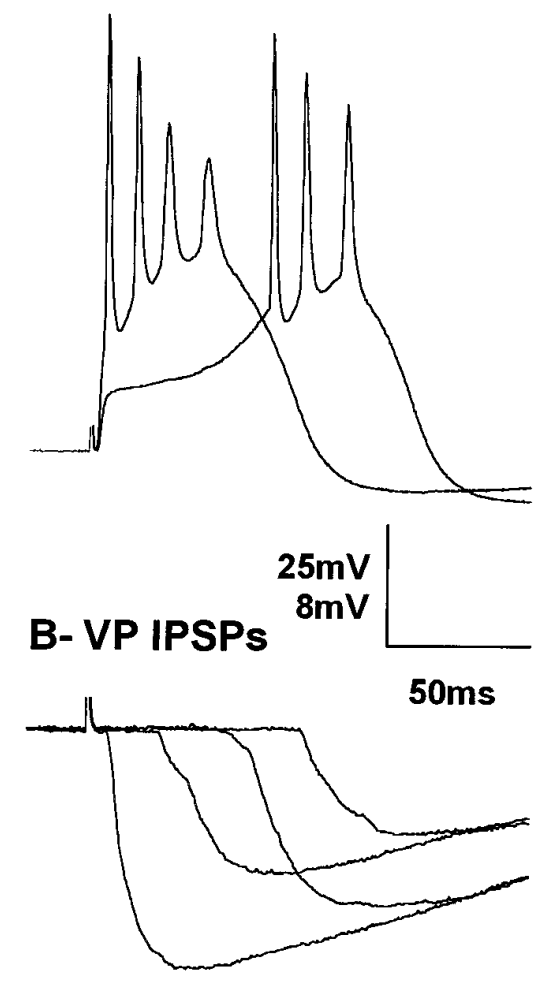

Figure 9. A, Current-clamp recording of an RTN neuron from a P10 animal, responding to stimulation of the internal capsule at two different intensities. $B$, Current-clamp recording of IPSPs in a VPN neuron recorded in the same slice as in $A$ showing four responses to different stimulus strengths. Vertical bar: $A, 25 \mathrm{mV} ; B, 8 \mathrm{mV}$. Horizontal bar: $A, B$, $50 \mathrm{msec}$.

of the nucleus injected are now elongated and commonly have tufts of secondary dendrites arising from primary dendrites located at each pole of an oval soma. The number of dendritic spines has become less, the remaining protrusions being less spine-like and more like excrescences.

VPN cells at P14 show a remarkable change from P10, suddenly acquiring a morphology not unlike that of the adult (Fig. 12B). Somata are larger, and the dendritic fields much more extensive. Many of the dendritic fields are radially symmetrical, but those possessed by cells close to the external medullary lamina can be flattened and elongated, as is typical of the adult. The number of dendritic branches still appears greater than in the adult, but the size difference between primary and secondary branches seen at all earlier ages is reduced. Secondary and tertiary branches are also much longer than at earlier ages. An occasional dendritic spine is seen, but the numbers are much smaller than on RTN cells at this age.

At P21, RTN cells are completely adult-like, with oval somata, very lengthy proximal dendrites often extending from the two poles of an oval soma and with most branching occurring toward the peripheral part of the dendritic field (Fig. 12C). Dendritic spines have now been lost. At the same age, VPN neurons have also acquired most of their adult characteristics, especially the typical symmetrical dendritic field and the bushy branching pattern of the primary dendrites (Fig. 12D).

Axons. Axons filled with dye could be identified arising from some injected RTN and VPN neurons at all ages (Figs. 10E, 11, 12) but in no case could they be traced for more than a few hundred microns from the soma, and no branches or terminals were filled.

\section{Quantitative observations}

Figures 13 and 14 show the results of the various types of measurements made on the populations of injected RTN and VPN cells from P3 to P21. These results confirm the pattern of maturation described above, with a number of additional facts.

From the measurements of area, perimeter, and diameter (Fig. $13 A-C, E-G)$, it is clear that the somata of both RTN and VP cells undergo a small increase in size from P3 to P7 but then at P10, undergo a significant reduction; this is greater in the RTN cells than in the VPN cells. For the RTN cells and, to a lesser extent, the VPN cells, this is the time at which the points of origins of dendrites from the soma are becoming more clearly defined and the outlines of the somata more discretely delineated. In both RTN and VPN cells after P10, there is a quite precipitate increase in soma size, which is then maintained to P21 and continues into adulthood (R. Warren and E. Jones, unpublished observations).

The form factor calculations (Fig. $13 D, H$ ) show that the somata of both RTN and VPN cells are quite round (form factor close to 1) at P3 and that this is maintained by VPN cells throughout the P3-P21 period. The somata of RTN cells at P7 undergo a change to a more ovoid profile (form factor $\leq 0.6$ ), which recovers over the P7-P14 period. At P14 and P21, the somata are as round as those of VPN cells, so the elongated appearance typical of the RTN cells at these ages and in the adult is probably conferred primarily by the elongated arrangement of the dendrites.

The more highly branched character of the dendrites of immature RTN and VPN cells and the progressive lengthening of the dendrites as the cells mature are evident in the graphs of dendritic intersections (Fig. 14A,B). The number of dendritic branches close to the soma is high in RTN neurons from $\mathrm{P} 3$ to P10 but then declines beyond P10 (Fig. 14A). As the dendrites lengthen with age, more branches are added distally, but the increase in distal dendritic branches is small and does not offset the large decrease in proximal branches, many of which are, therefore, presumably lost.

VPN cells (Fig. 14B) show a progressive increase in proximal dendritic branches that moves in a distal direction as the dendrites grow from P10 to P21. More distal branches are added at P14 and $\mathrm{P} 21$. The appearance suggests continuous growth rather than an overproduction and later loss of dendritic branches.

The mean extent of dendritic fields measured for RTN and VPN neurons showed parallel linear growth between P3 and P21 (Fig. 14C). The mean extent of the dendritic field, which was $89 \pm 7 \mu \mathrm{m}$ and $90 \pm 8 \mu \mathrm{m}$ for RTN and VPN neurons at $\mathrm{P} 3$, increased by a factor of 2.6 to $232 \pm 20 \mu \mathrm{m}$ and $236 \pm$ $10 \mu \mathrm{m}$, respectively, at $\mathrm{P} 21$. In addition, the general shape of the dendritic field changed but only in RTN neurons. Whereas the dendrites appeared to radiate from the soma in the majority of RTN and VPN neurons at P3, that general pattern remained constant in VPN neurons, but RTN neurons became polarized with development, the large majority of them ending with dendrites running parallel to the long, approximately dorso-ventral axis of the nucleus. In some VPN neurons, the large primary dendrites originated at opposite ends of the soma but then tended to radiate from there like a fan (Figs. 12B, $14 D$ ). By contrast, dendrites of RTN neurons ran in parallel. 


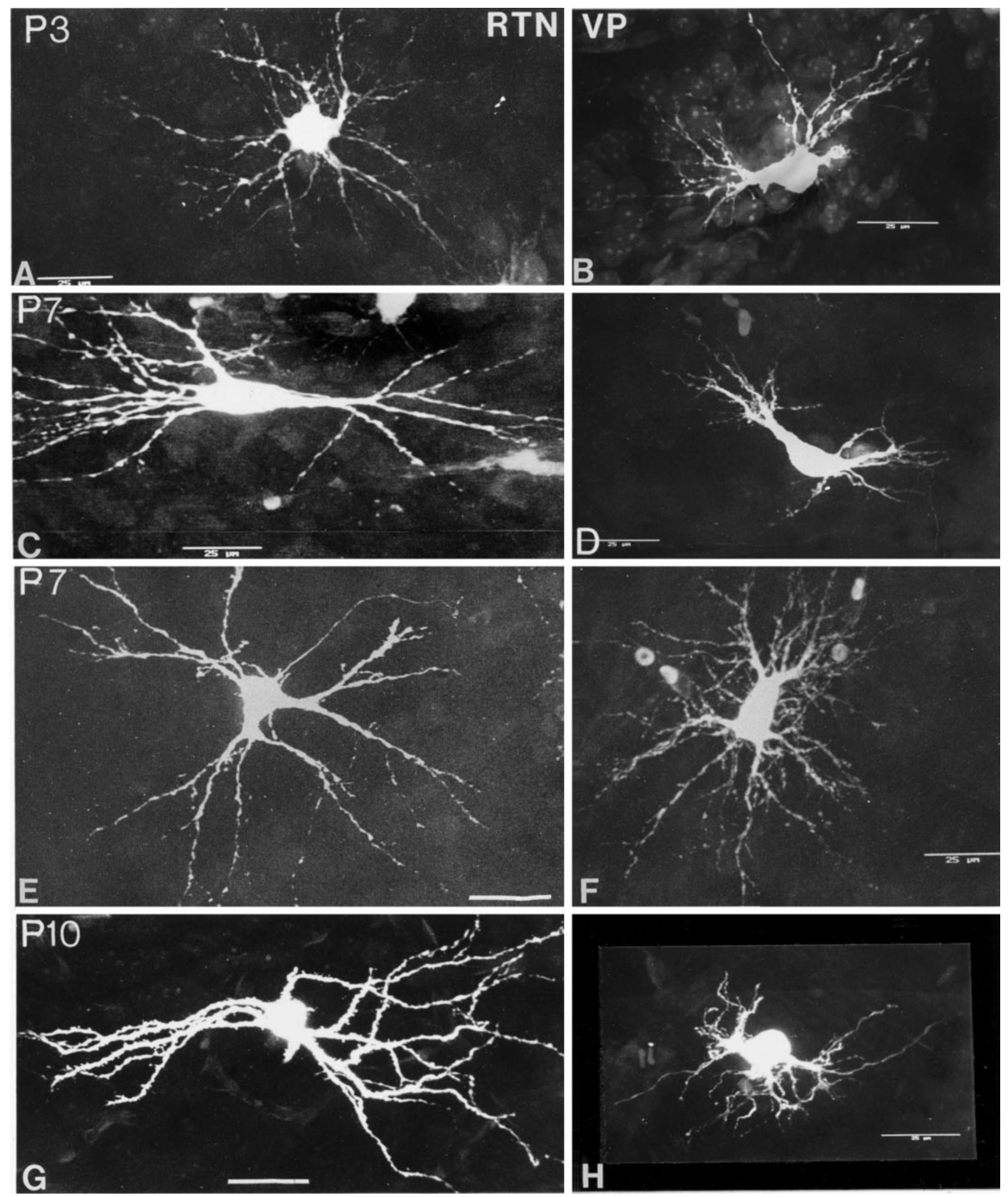

Figure 10. Confocal images of Lucifer yellow-filled RTN (left column) and VPN neurons (right column) from P3 to P14. Cells immunoreactive for GABA are faintly visible in the background of RTN in some of the images. Note the accelerated maturation of the RTN neurons in comparison with the VPN neurons from P7. Scale bars, $25 \mu \mathrm{m}$. 


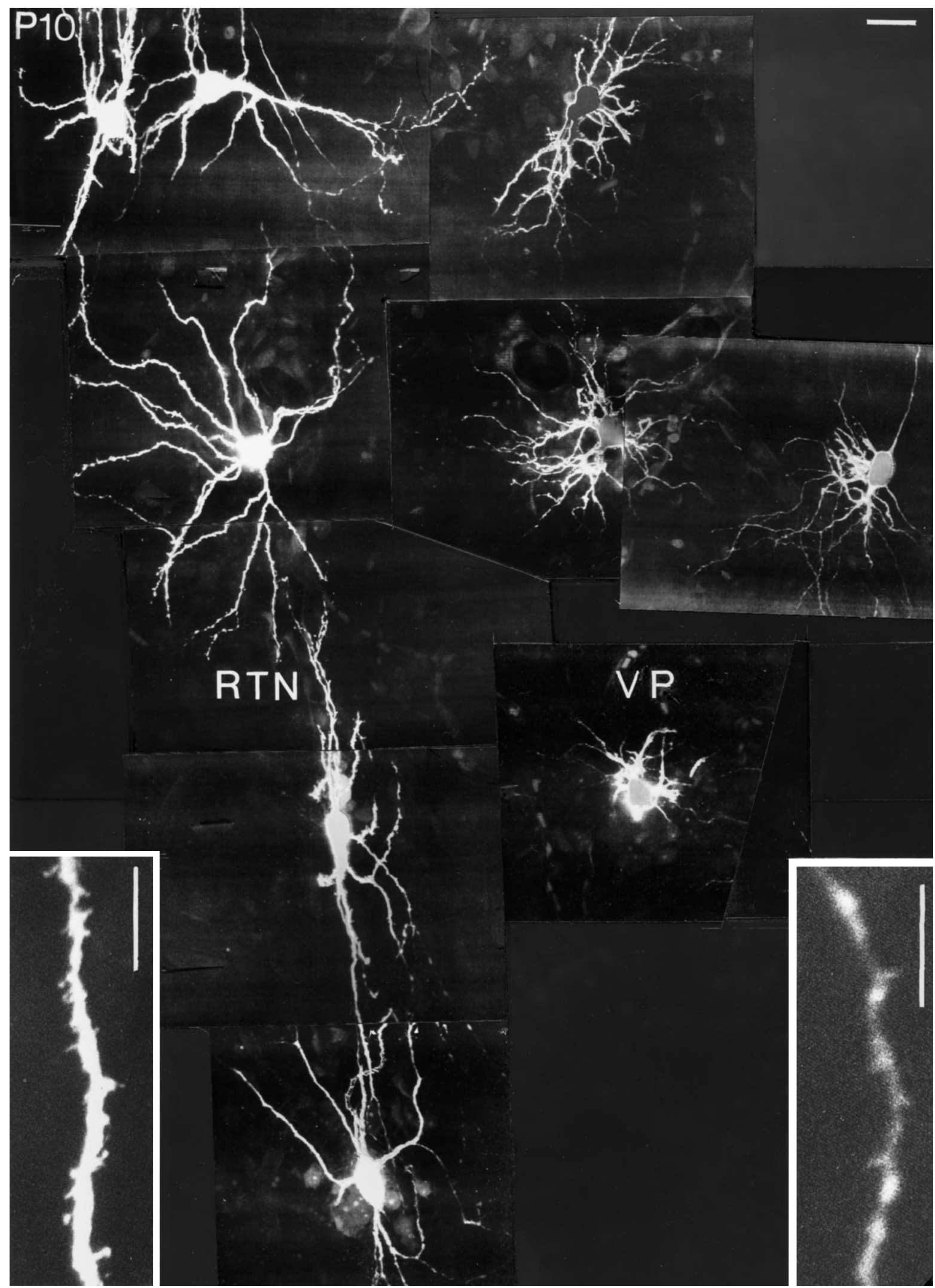

Figure 11. Confocal images of Lucifer yellow-filled RTN (left) and VPN (right) neurons in the same slice at P10. Note the immaturity of the VPN neurons compared with the RTN neurons. Scale bars, $25 \mu \mathrm{m}$. 


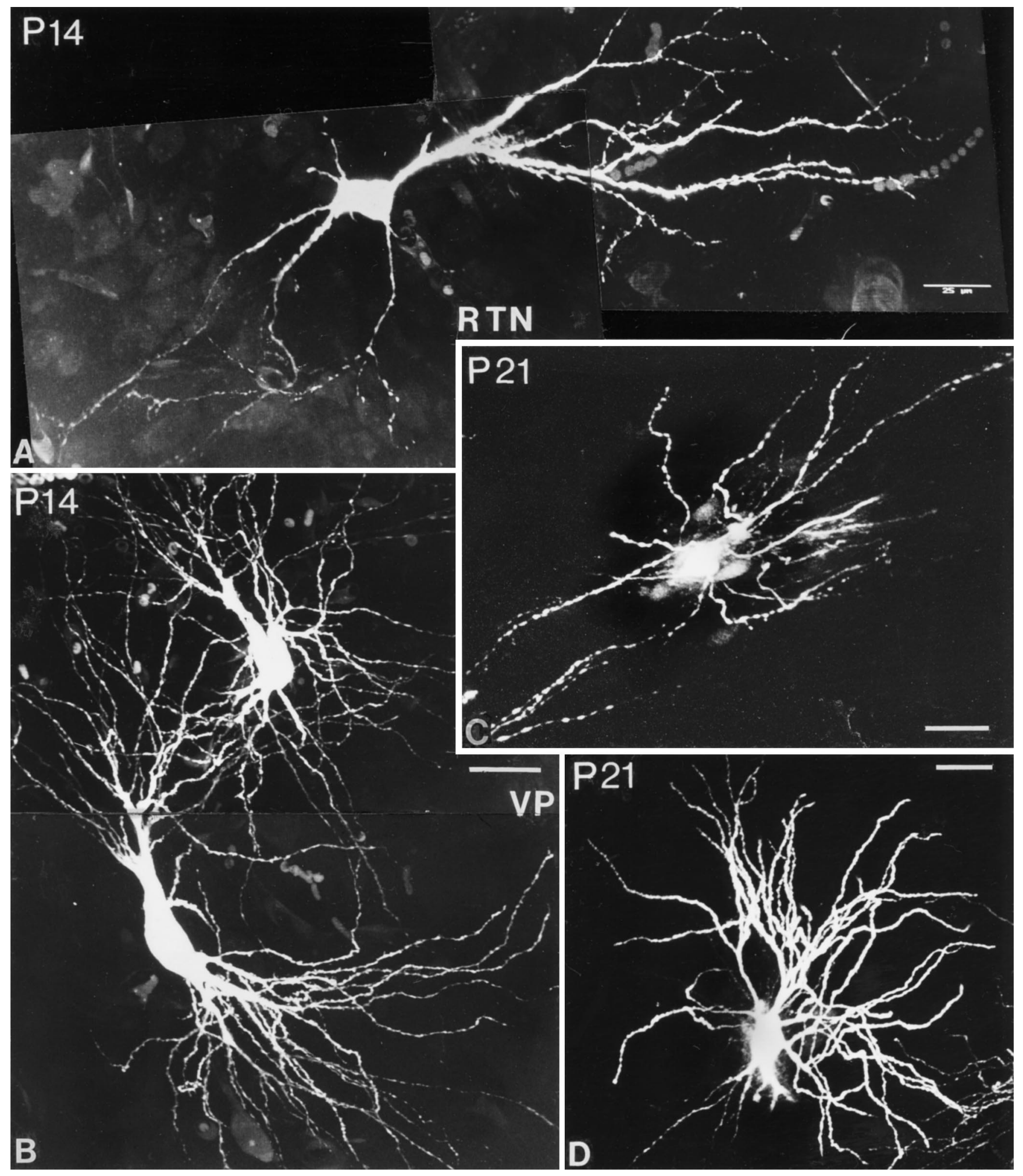

Figure 12. Confocal images of Lucifer yellow-filled RTN $(A, C)$ and VPN $(B, D)$ cells at P14 and P21. RTN cells have their mature form at P14. In $C$, dendrites have been cut off in formatting the plate. VPN cells have begun to mature at P14 (B), but morphogenesis is only complete at P21 $(D)$. One major dendrite trunk has been cut off in $D$. Scale bars, $25 \mu \mathrm{m}$.

Sometimes they radiated from the soma, but within $10-20 \mu \mathrm{m}$, the orientation of many changed so that they became parallel to the long axis of the nucleus for most of their length (Figs. $12 C, 14 D)$.

\section{DISCUSSION}

Low-frequency spindle-like oscillations can be evoked by single electrical shocks of the internal capsule in in vitro mouse thalamocortical 


\section{RTN}

A- Area

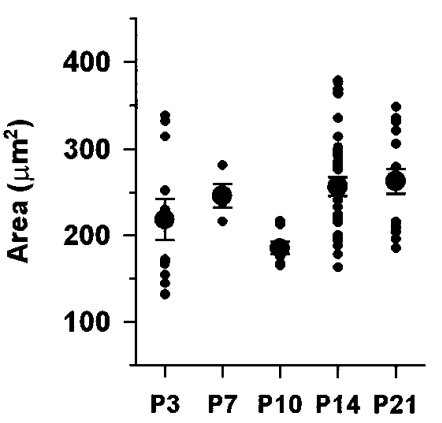

C- Mean diameter

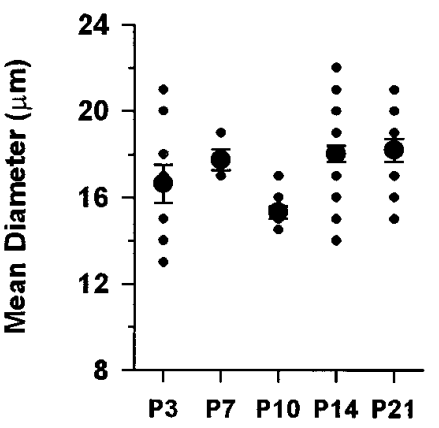

Postnatal Age (days)
B- Perimeter

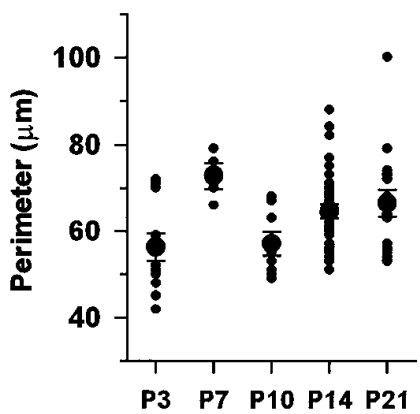

D- Form factor

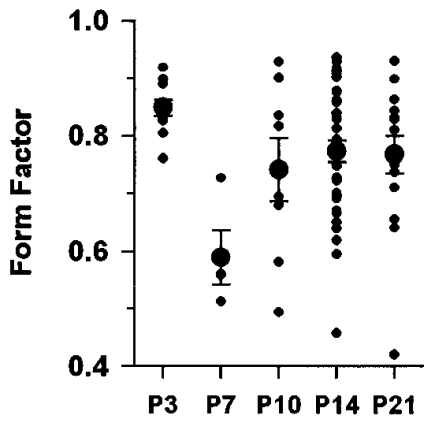

Postnatal Age (days)
E- Area

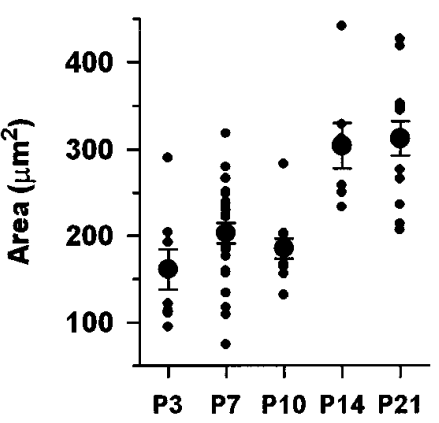

G- Mean diameter

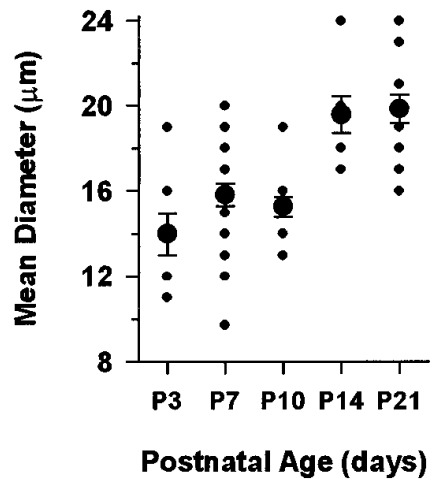

F- Perimeter

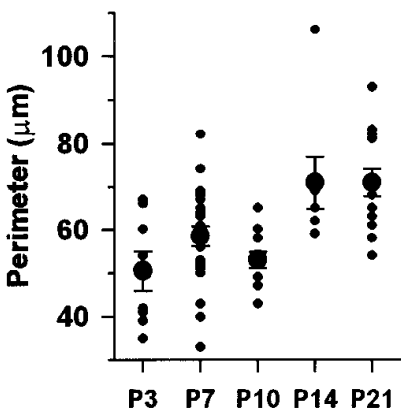

H- Form factor

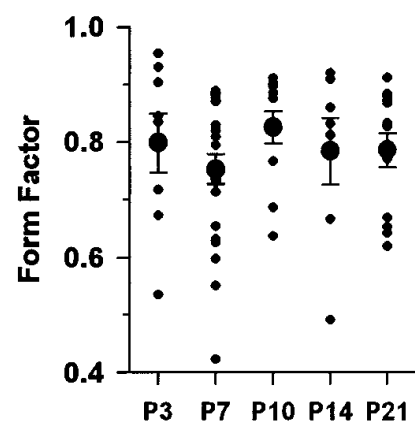

Postnatal Age (days)

Figure 13. Postnatal development of RTN (left, $A-D)$ and VPN (right, $E-H)$ neuronal somata. Measurements were made from confocal images of Lucifer yellow-filled neurons in the immunocytochemically identified RTN. Small solid circles represent the value from single neurons, and large solid circles with error bars show the average \pm SEM. The number of RTN neurons measured for each age was P3, 11; P7, 4; P10, 8; P14, 34; P21, 15; and the total number was 72. The number of VPN neurons for each age was P3, 8; P7, 23; P10, 11; P14, 7; P21, 13; and the total number was 62.

slices from P12 to P21 (Warren et al., 1994). These oscillations are engendered through synaptic interactions between RTN and VP neurons and depend on the ability of these neurons to fire bursts of multiple $\mathrm{Na}^{+} / \mathrm{K}^{+}$action potentials supported by LTS. Comparable oscillations can be elicited in VPN of rat slices by stimulation of RTN (Huguenard and Prince, 1994) and are generated spontaneously in slices of ferret dorsal lateral geniculate nucleus (von Krosigk et al., 1993; Bal et al., 1995a, b). In vivo rhythmic burst firing of RTN induces spindle oscillations in dorsal thalamic nuclei (Steriade et al., 1985, 1993). The present study followed the development of oscillations in P3-P11 mice and examined morphological characteristics of injected RTN and VPN neurons between P3 and P21. Functional synaptic connections were present at P3-P4, but intrathalamic oscillations were not observed before P12. Appearance of oscillations at P12 was attributable to maturation of membrane and synaptic characteristics converging at this time point. Physiological maturation of RTN and VPN neurons was paralleled by rapid morphological changes between P10 and P14, with RTN neurons leading VPN neurons.

\section{Intrinsic properties}

Although the physiological properties of thalamic neurons were markedly immature in comparison with older animals (Warren et al., 1994), relative differences between RTN and VPN neurons could be detected from P3, showing that RTN and VPN neurons are physiologically differentiated at this age. Postnatal changes in membrane properties, including increasingly more negative RMP, shortening of $\tau_{\mathrm{m}}$, decrease in $\mathrm{R}_{\mathrm{in}}$, and shortening of $\mathrm{Na}^{+} / \mathrm{K}^{+}$ action potential duration, occurred in parallel in RTN and VPN neurons and were similar to neurons in lateral geniculate and perigeniculate nuclei (White and Sur, 1992; Ramoa and McCormick, 1994; McCormick et al., 1995) and in cerebral cortex, (McCormick and Prince, 1987) and hippocampus (Zhang et al., 1991).

Lowering of RMP to more negative values could be attributable partly to increase in $\mathrm{Na}^{+} / \mathrm{K}^{+}$ATPase activity during postnatal development (Fukuda and Prince, 1992) associated with increased $\mathrm{K}^{+}$conductance (Spigelman et al., 1992) and/or decreased $\mathrm{Cl}^{-}$ permeability (Zhang et al., 1991). Because RMP of RTN neurons became negative relative to the activation potential of the LTS, initiation of spindle oscillations in RTN cells likely occurs only through an initial depolarization; moreover, rebound bursts after the afterhyperpolarization will only be generated during a spindle sequence when RTN neurons are significantly depolarized (see Steriade and Deschênes, 1988; Steriade et al., 1990).

The increase in soma size and substantial elaboration of dendritic arborizations indicates that the membrane surface of RTN and VPN cells expands considerably in the neonatal period. (The dip in soma size of RTN neurons at P10 is probably of little significance, simply reflecting the more defined points of origin of primary dendrites.) Increase in cell size could account in large 


\section{A- RTN Dendritic Intersections}

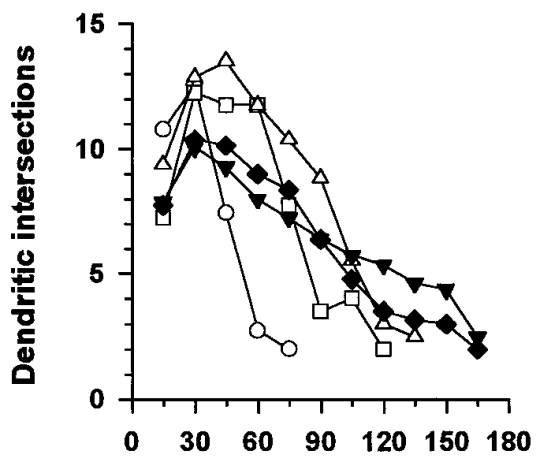

\section{B- VP Dendritic Intersections}

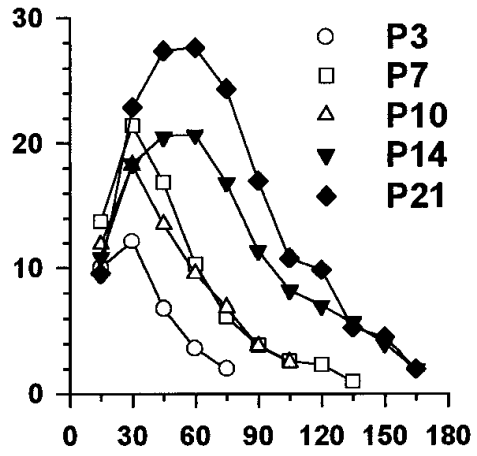

Distance from soma centre ( $\mu \mathrm{m})$

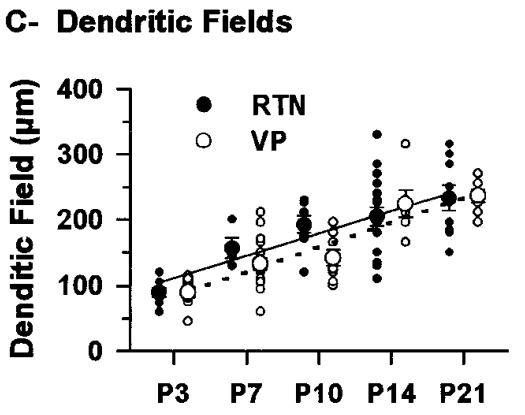

Postnatal Age (days)

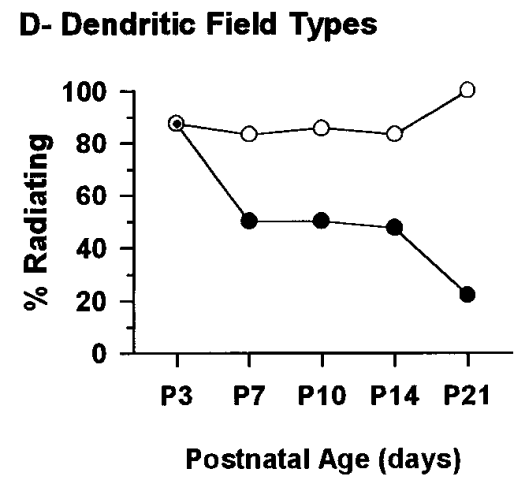

Figure 14. $A, B$, Plots of the number of dendritic branches as a function of the distance from the soma for RTN and VPN neurons at different ages. Measurements were made from confocal images similar to those shown in Figures 10-12. The dendritic branches were counted every $15 \mu \mathrm{m}$, starting at the center of the soma and up to a distance of $330 \mu \mathrm{m}$. Each point represents the average of several neurons. The number of neurons for RTN was P3, 9; P7, 4; P10, 8; P14, 18; P21, 8; and the total number was 47 . The number of VPN neurons was P3, 9; P7, 18; $\mathrm{P} 10,8 ; \mathrm{P} 14,6$; $\mathrm{P} 21,8$; and the total number was $49 . C, D$, Diameter and form of the dendritic field of RTN and VPN neurons. $C$, The extent of the dendritic field of RTN and VPN measured close to its larger axis with the soma in the center. Small circles represent the value for individual neurons, and the large circles with error bars show the average \pm SEM. $D$, Percentages of RTN and VPN neurons with their dendritic field radiating from the soma as opposed to those having their dendrites running parallel to the longer axis of the soma. The number of RTN neurons was $\mathrm{P} 3,8 ; \mathrm{P} 7,4$; $\mathrm{P} 9,8 ; \mathrm{P} 21,19$; and the total number was 48. The number of VPN neurons was P3, 8; P7, 18; P10, 8; P14, 6; P21, 7; and the total number was 47. part for the decrease in $\mathrm{R}_{\text {in }}$ observed in RTN and VPN. The concomitant decrease in $\tau_{\mathrm{m}}$ suggests that specific membrane resistivity decreases significantly, because specific membrane capacitance is likely to remain constant (Hille, 1984; Johnston and Wu, 1992). RTN neurons had smaller somata than VPN neurons and about half the number of dendritic branches but displayed $\tau_{\mathrm{m}}$ values much lower than VPN neurons, suggesting a much lower specific membrane resistivity. The decrease in membrane resistivity likely reflects increasing density of ion channels during postnatal development (c.f., McCormick and Prince, 1987; Spigelman et al., 1992). The increased inward rectification suggests a postnatal increase in the hyperpolarization-activated cation current $I_{\mathrm{h}}$. $I_{\mathrm{h}}$ probably plays a significant role in generation of delta waves through interplay with the LTS (McCormick and Pape, 1991; Steriade et al., 1993). Postnatal maturation of $I_{\mathrm{hr}}$ will contribute to the full spectrum of oscillations observable in the thalamocortical system, but it is not yet clear whether it has a role in the postinhibitory rebounds responsible for the low-frequency oscillations examined in the present study.

The most profound changes in membrane and morphological properties occurred during the first two postnatal weeks, before the appearance of intrathalamic oscillations. The key change was a sudden increase in the capacity of the LTS to generate multiple $\mathrm{Na}^{+} / \mathrm{K}^{+}$action potentials in RTN neurons that were then able to produce IPSPs in VPN neurons sufficiently large to deinactivate the LTS. The capacity of perigeniculate neurons to generate multiple action potentials from LTS spikes is also limited in ferrets before P30, with comparable lack or weakness of spindle oscillations in connected perigeniculate-dorsal lateral geniculate neurons between P22 and P30 (McCormick et al., 1995). In mice, LTS were present by P3-P4 but often failed to generate $\mathrm{Na}^{+} / \mathrm{K}^{+}$ actions potentials in VPN neurons before P9 and could generate few or none in RTN neurons. Lack of bursting was probably a consequence of the small size of the LTS (Pirchio et al., 1990; Ramoa and McCormick, 1994), which failed to reach $\mathrm{Na}^{+} / \mathrm{K}^{+}$ spike firing threshold, especially in VPN neurons in which the LTS activation potential is normally more negative than in RTN neurons (Huguenard and Prince, 1992; present results). Generation of $\mathrm{Na}^{+} / \mathrm{K}^{+}$spiking may also have been prevented by the offset of the LTS because of activation of subthreshold $\mathrm{K}^{+}$currents (Coulter et al., 1989; Crunelli et al., 1989; Hernández-Cruz and Pape, 1989; Huguenard et al., 1991; Budde et al., 1992; Pape et al., 1994).

During the first postnatal week, RTN and VPN neurons fired tonically when depolarized from RMP but started to fire in bursts at $\sim$ P8. These properties may be important for normal development of the thalamo-cortical system, which is in part activitydependent (for review, see Fox, 1995).

\section{Synaptic properties}

Electrical stimulation of the internal capsule at P3-P4 evoked glutamatergic EPSPs in RTN neurons and IPSPs in VPN neurons, indicating that collaterals from corticothalamic and/or thalamocortical fibers have made functional contacts on RTN neurons and that synaptic connections between RTN and VPN cells have also formed. Although synaptic connections are present from at least P3-P4, RTN neurons only begin to fire bursts in response to stimulation of the internal capsule at P8 when the RMP of the neurons falls below LTS activation potential. From P8 to P10, the latency between EPSP and burst depended on stimulus strength, so that RTN neurons did not fire simultaneously. From P11, firing latency variations in RTN neurons were much smaller, ensuring 
synchronous bursting of simultaneously excited cells. Synchronous firing of populations of RTN neurons is probably a key event in the induction of thalamic oscillations (Steriade et al., 1985, 1993; Bal et al., 1995b).

RTN-induced IPSPs in young VP neurons were mediated by $\mathrm{GABA}_{\mathrm{A}}$ receptors. In ferret lateral geniculate nucleus, a $\mathrm{GABA}_{\mathrm{B}}$ response was uncovered only when $\mathrm{GABA}_{\mathrm{A}}$ receptors were blocked (von Krosigk et al., 1993; Bal et al., 1995a,b; Destexhe and Sejnowski, 1995). In mice, BMI significantly prolonged synaptically evoked bursts in RTN neurons, but this was insufficient to uncover GABA $_{\mathrm{B}}$ IPSPs despite presence of functional $\mathrm{GABA}_{\mathrm{B}}$ receptors in mouse thalamus (Lin et al., 1993; Caddick and Hosford, 1996).

\section{Morphological characteristics}

The maturation of RTN and VP neurons followed a sequence typical of all CNS neurons, with progressive growth accompanied by generation of an excess of dendritic branches and spine-like protrusions that were subsequently retracted. Somal size increased by $100 \%$ and dendritic field size by $>250 \%$ in both cell types. A similar pattern of morphogenesis has been described in Golgi-stained dorsal lateral geniculate nucleus and after retrograde labeling of geniculo-cortical cells (Parnavelas et al., 1977; Mason, 1983; Dalva et al., 1994; Sutton and Brunso-Bechtold, 1991). Development of RTN cells has not been described. RTN cells undergo considerable remodeling, particularly of dendritic fields, over a short, defined time span, following a program independent of that of VPN cells. The physiological results showed that synapse formation has occurred and effective synaptic function established in RTN and VPN cells well before maturation of their definitive form, although we cannot rule out that density and distribution of synapses change in association with alterations in dendritic architecture and may, indeed, be one of the major impetuses to dendritic remodeling. RTN neurons are advanced over VPN cells, undergoing a sudden abrupt change in form at P7 and acquiring their adult form between P10 and P14. VPN neurons undergo a similar growth spurt, but at P10, so that their maturation is delayed in comparison with RTN cells, acquiring the adult form only by P21. The program of maturation of VPN cells appears to be synchronous with the increase in complexity and density of terminal branching of their axons within the barrels of somatosensory cortex, which peaks at P12 (Agmon et al., 1993). There have been no studies to determine whether growth in complexity of dendritic trees of RTN and VPN neurons is associated with increasing density of afferent connections. In dorsal lateral geniculate nucleus (Dalva et al., 1994), dendritic growth proceeds independently of afferent innervation.

Rapid maturation of RTN and VPN cells in the second postnatal week occurs in parallel with maturation of the network to the extent that it can support slow oscillations. Although as revealed here, these oscillations are largely dependent on membrane properties, establishment of the definitive morphologies of the cells is likely (see above) to contribute to some of these properties, as well as to reflect changes in densities and distributions of synapses. These features, which should facilitate activation of ensembles of RTN and VPN cells may also be factors in establishing oscillatory activity in the network, with its behavioral concomitants of sleep and wakefulness. Immaturity in the network and lack of a cellular basis for spindle oscillations may also underlie the failure of spike and wave phenomena typical of absence seizures to develop in children before 4-6 years of age (Aicardi, 1994).
Note added in proof: Since submitting this paper, we have been able to consistently demonstrate $G A B A_{B}$-mediated IPSPs in slices from $P 3$ and older mice.

\section{REFERENCES}

Agmon A, Connors BW (1991) Thalamocortical responses of mouse somatosensory (barrel) cortex in vitro. Neuroscience 41:365-379.

Agmon A, O'Dowd DK (1992) NMDA receptor-mediated currents are prominent in the thalamocortical synaptic response before maturation of inhibition. J Neurophysiol 68:345-349.

Agmon A, Yang LT, O'Dowd DK, Jones EG (1993) Organized growth of thalamocortical axons from the deep tier of terminations into layer IV of developing mouse barrel cortex. J Neurosci 13:5365-5382.

Agmon A, Yang LT, Jones EG, O'Dowd DK (1995) Topological precision in the thalamic projection to neonatal mouse barrel cortex. J Neurosci 15:549-561.

Aicardi J (1994) Epilepsy in children. New York: Raven.

Bal T, von Krosigk M, McCormick DA (1995a) Synaptic and membrane mechanisms underlying synchronized oscillations in the ferret lateral geniculate nucleus in vitro. J Physiol (Lond) 483:641-663.

Bal T, von Krosigk M, McCormick DA (1995b) Role of the ferret perigeniculate nucleus in the generation of synchronized oscillations in vitro. J Physiol (Lond) 483:665-685.

Budde T, Mager R, Pape H-C (1992) Different types of potassium outward current in relay neurons acutely isolated from the rat lateral geniculate nucleus. Eur J Neurosci 4:708-722.

Caddick SJ, Hosford DA (1996) $\mathrm{GABA}_{\mathrm{B}}$-activated $\mathrm{gK}^{+}$in thalamic neurons in the lethargic $(l h / l h)$ mouse model of generalized absence seizures. Neurosci Lett 205:29-32.

Catalano SM, Robertson RT, Killackey HP (1995) Rapid alteration of thalamocortical axon morphology follows peripheral damage in the neonatal rat. Proc Natl Acad Sci USA 92:2549-2552.

Coulter DA, Huguenard JR, Prince DA (1989) Calcium currents in rat thalamocortical relay neurones: kinetic properties of the transient, low-threshold current. J Physiol (Lond) 414:587-604.

Crunelli V, Lightowler S, Pollard CE (1989) A T-type $\mathrm{Ca}^{2+}$ current underlies low-threshold $\mathrm{Ca}^{2+}$ potentials in cells of the cat and rat lateral geniculate nucleus. J Physiol (Lond) 413:543-561.

Dalva MB, Ghosh A, Shatz CJ (1994) Independent control of dendritic and axonal form in the developing lateral geniculate nucleus. J Neurosci 14:3588-3602.

Deschênes M, Madariaga-Domich A, Steriade M (1985) Dendrodendritic synapses in the cat reticularis thalami nucleus: a structural basis for thalamic spindle synchronization. Brain Res 334:165-168.

Destexhe A, Sejnowski TJ (1995) G-protein activation kinetics and spillover of $\gamma$-aminobutyric acid may account for differences between inhibitory responses in the hippocampus and thalamus. Proc Natl Acad Sci USA 92:9515-9519.

Domich L, Oakson G, Deschênes M, Steriade M (1987) Thalamic and cortical spindles during early ontogenesis in kittens. Brain Res $31: 140-142$.

Fox K (1995) A critical period for long-term potentiation in primary sensory cortex. Neuron 15:485-488.

Fukuda A, Prince DA (1992) Postnatal development of electrogenic sodium pump activity in rat hippocampal pyramidal neurons. Dev Brain Res 65:101-114.

Harper RM (1983) Infant sleep development. In: Sleep mechanisms and functions in humans and animals: an evolutionary perspective (Meyes A, ed), pp 107-125. Wokingham, UK: Van Nostrand Rheinhold.

Hernández-Cruz A, Pape H-C (1989) Identification of two calcium currents in acutely dissociated neurons from the rat lateral geniculate nucleus. J Neurophysiol 61:1270-1283.

Hille B (1984) Ion channels of excitable membranes. Sunderland, MA: Sinauer.

Houser CR, Vaughn JE, Barber RP, Roberts E (1980) GABA neurons are the major cell type of the nucleus reticularis thalami. Brain Res 200:341-354.

Huguenard JR, Prince DA (1992) A novel T-type current underlies prolonged $\mathrm{Ca}^{2+}$-dependent burst firing in GABAergic neurons of rat thalamic reticular nucleus. J Neurosci 12:3804-3817.

Huguenard JR, Prince DA (1994) Intrathalamic rhythmicity studied in vitro: nominal T-current modulation causes robust anti-oscillatory effects. J Neurosci 14:5485-5502. 
Huguenard JR, Coulter DA, Prince DA (1991) A fast transient potassium current in thalamic relay neurons: kinetics of activation and inactivation. J Neurophysiol 66:1304-1315.

Jahnsen H, Llinás R (1984) Ionic basis for the electroresponsiveness and oscillatory properties of guinea-pig thalamic neurones in vitro. J Physiol (Lond) 349:227-247.

Johnston D, Wu SM-S (1995) Foundations of cellular neurophysiology. Cambridge, MA: MIT.

Jones EG, Huntley GW, Benson DL (1994) Alpha calcium/calmodulindependent protein kinase II selectively expressed in a subpopulation of excitatory neurons in monkey sensory-motor cortex: comparison with GAD-67 expression. J Neurosci 14:611-629.

Jouvet-Mounier D, Astic L, Lacotte D (1970) Ontogenesis of the states of sleep in rat, cat, and guinea pig during the first postnatal month. Dev Psychobiol 2:216-239.

Lin F-H, Cao Z, Hosford DA (1993) Selective increase in GABA $_{\mathrm{B}}$ receptor number in lethargic $(l h / l h)$ mouse model of absence seizures. Brain Res 608:101-106.

Mason CA (1983) Postnatal maturation of neurons in the cat's lateral geniculate nucleus. J Comp Neurol 217:458-469.

McCormick DA (1991) Functional properties of a slowly inactivating potassium current in guinea pig dorsal lateral geniculate relay neurons. J Neurophysiol 66:1176-1189.

McCormick DA, Pape H-C (1991) Properties of a hyperpolarizationactivated cation current and its role in rhythmic oscillation in thalamic neurones. J Physiol (Lond) 431:291-318.

McCormick DA, Prince DA (1987) Post-natal development of electrophysiological properties of rat cerebral cortical pyramidal neurones. $\mathbf{J}$ Physiol (Lond) 393:743-762.

McCormick DA, Trent F, Ramoa AS (1995) Postnatal development of synchronized network oscillations in the ferret dorsal lateral geniculate and perigeniculate nuclei. J Neurosci 15:5739-5752.

Parnavelas JG, Mounty EJ, Bradford R, Lieberman AR (1977) The postnatal development of neurons in the dorsal lateral geniculate nucleus of the rat: a Golgi study. J Comp Neurol 171:481-500.

Pape H-C, Budde T, Mager R, Kisvárday ZF (1994) Prevention of $\mathrm{Ca}^{2+}$ mediated action potentials in GABAergic local circuit neurones of rat thalamus by a transient $\mathrm{K}^{+}$current. J Physiol (Lond) 478:403-422.

Pirchio M, Lighttowler S, Crunelli V (1990) Postnatal development of the T calcium current in cat thalamocortical cells. Neuroscience 38:39-45.

Ramoa AS, McCormick DA (1994) Developmental changes in electrophysiological properties of LGNd neurons during reorganization of retinogeniculate connections. J Neurosci 14:2089-2097.

Sholl DA (1953) Dendritic organization in the neurons of the visual and motor cortices of the cat. J Anat 87:387-306.
Soltesz I, Lightowler S, Leresche N, Jassik-Gerschenfeld D, Pollard CE, Crunelli V (1991) Two inward currents and the transformation of low-frequency oscillations of rat and cat thalamocortical cells. J Physiol (Lond) 441:175-197.

Spigelman I, Zhang L, Carlen PL (1992) Patch-clamp study of postnatal development of CA1 neurons in rat hippocampal slices: membrane excitability and $\mathrm{K}^{+}$currents. J Neurophysiol 68:55-69.

Steriade M, Deschênes M (1988) Intrathalamic and brainstem-thalamic networks involved in resting and alert states. In: Cellular thalamic mechanisms (Bentivoglio M, Spreafico R, eds), pp 51-76. Amsterdam: Elsevier.

Steriade M, Deschênes M, Domich L, Mulle C (1985) Abolition of spindle oscillations in thalamic neurons disconnected from nucleus reticularis thalami. J Neurophysiol 54:1473-1497.

Steriade M, Jones EG, Llinás RR (1990) Thalamic oscillations and signaling. New York: Wiley.

Steriade M, McCormick DA, Sejnowski TJ (1993) Thalamocortical oscillations in the sleeping and aroused brain. Science 262:679-685.

Sterman MB (1972) The basic rest-activity cycle and sleep: developmental considerations in man and cats. In: Sleep and the maturing nervous system (Clemente CD, Purpura DP, Mayer FE, eds), pp 175-197. New York: Academic.

Sutton JK, Brunso-Bechtold JK (1991) A Golgi study of dendritic development in the dorsal lateral geniculate nucleus of the normal ferrets. J Comp Neurol 309:71-85.

von Krosigk M, Bal T, McCormick DA (1993) Cellular mechanisms of a synchronized oscillation in the thalamus. Science 262:679-685.

Warren RA, Jones EG (1994) Postnatal development of physiological and anatomical characteristics of thalamic ventroposterior and reticular neurons. Soc Neurosci Abstr 20:25.

Warren RA, Jones EG (1995) Postnatal development of inhibitory postsynaptic potentials in the nucleus ventroposterior of the thalamus. IBRO Abstr 4:409.

Warren RA, Agmon A, Jones EG (1994) Oscillatory synaptic interactions between ventroposterior and reticular neurons in mouse thalamus in vitro. J Neurophysiol 72:1993-2003.

White CA, Sur M (1992) Membrane and synaptic properties of developing lateral geniculate nucleus neurons during retinogeniculate axon segregation. Proc Natl Acad Sci USA 89:9850-9854.

Yen CT, Conley M, Hendry SHC, Jones EG (1985) The morphology of physiologically identified GABAergic neurons in the somatic sensory part of the thalamic reticular nucleus in the cat. J Neurosci 5:2254-2268.

Zhang L, Spigelman I, Carlen PL (1991) Development of GABAmediated, chloride-dependent inhibition in CA1 pyramidal neurones of immature rat hippocampal slices. J Physiol (Lond) 444:25-49. 